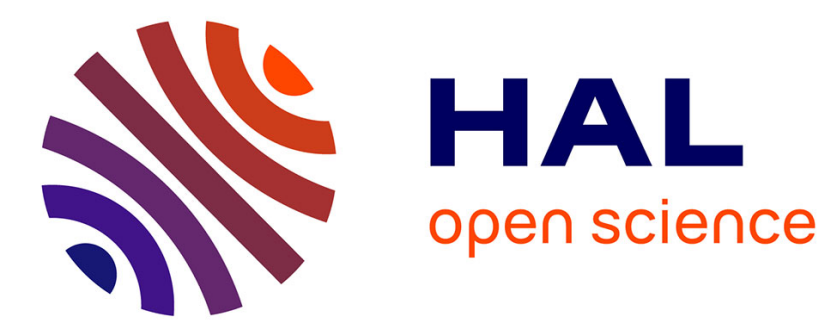

\title{
Le transport ferroviaire régional de voyageurs : un processus collectif d'apprentissage
}

Yves Crozet, Christian Desmaris

\section{To cite this version:}

Yves Crozet, Christian Desmaris. Le transport ferroviaire régional de voyageurs : un processus collectif d'apprentissage. RTS - Recherche Transports Sécurité, 2011, 27 (3), pp.143-162. 10.1007/s13547-0110015-3 . halshs-00601981

\section{HAL Id: halshs-00601981 https://shs.hal.science/halshs-00601981}

Submitted on 14 Oct 2011

HAL is a multi-disciplinary open access archive for the deposit and dissemination of scientific research documents, whether they are published or not. The documents may come from teaching and research institutions in France or abroad, or from public or private research centers.
L'archive ouverte pluridisciplinaire HAL, est destinée au dépôt et à la diffusion de documents scientifiques de niveau recherche, publiés ou non, émanant des établissements d'enseignement et de recherche français ou étrangers, des laboratoires publics ou privés. 


\title{
Le transport ferroviaire régional de voyageurs : un processus collectif d'apprentissage
}

\author{
The regional rail passenger transport : \\ a collective learning process
}

\section{Publié dans la revue Recherche Transports Sécurité (RTS), numéro spécial sur le transport ferroviaire en France et dans le monde, vol 27, n³, 2011.}

\author{
Yves CROZET et Christian DESMARIS \\ Université de Lyon - Institut d'Etudes Politiques \\ Laboratoire d’Économie des Transports (UMR n5593 www.let.fr) \\ yvescrozet@sciencespo-lyon.fr, christian.desmaris@sciencespo-lyon.fr
}

\section{Résumé}

Le transport ferroviaire régional de voyageurs se présente depuis la fin des années 90 comme une sorte d'exception française. C'est en effet un secteur où une réforme a été conduite sereinement et a connu un réel succès, sans doute car elle a été progressive et a pris le temps de l'expérimentation. Ainsi, après les 6, puis 7 régions expérimentatrices, qui se sont lancées en 1997 dans ce qui est encore un processus collectif d'apprentissage, ce sont l'ensemble des régions métropolitaines (Corse et Ile-de-France exceptées) qui sont entrées en janvier 2002 dans l’ère de la gestion décentralisée du transport régional de voyageurs.

Aujourd'hui, nous avons le recul nécessaire pour évaluer ce processus d'apprentissage, son cheminement, ses résultats et ses perspectives. Une évaluation qui est, dans un premier temps, rétrospective, pour éclairer le jeu des acteurs et la nouvelle dynamique qui en a découlé, tant pour l'offre que pour la demande. Nous proposons ensuite une évaluation prospective car le succès même de la régionalisation invite à poursuivre le processus d'apprentissage, fut-ce en s'approchant de transformations qui relèvent sans doute plus de la discontinuité que de la continuité.

Mots clés : apprentissage, transport ferroviaire de voyageurs, évaluation, régionalisation, TER

\begin{abstract}
Regional rail passenger services are prevalent in France, as opposed to the more marginal role they play elsewhere in the world. It is indeed at the regional level that reforms have been successfully implemented, probably because the process was gradual and cautious. Initially in 1997, seven regions began experimenting with a decentralized system of regional passenger transportation services. By 2002, all metropolitan regions, with the exception of Corsica and Ilede-France, were involved. Today we have reached a point where this process can be retrospectively and prospectively assessed. This article is first an assessment of the role of actors and the emerging dynamic between regional passenger rail transport supply and demand. Then, a prospective is offered since regionalization is best understood as an ongoing learning process that can be discontinuous or continuous.
\end{abstract}

Key words : Learning, passenger rail, assessment, regionalization 
La loi SRU ${ }^{1}$ qui généralise le transfert aux régions du transport régional de voyageurs a été votée il y a dix ans, en décembre 2000. Sa mise en application a commencé en janvier 2002. Elle généralisait une expérimentation lancée 3 années plus tôt dans 7 régions françaises. Ces expérimentations, un cas relativement rare en France, faisaient suite aux préconisations du rapport Haenel (1993), rédigé pour redonner un horizon au transport ferroviaire alors que se dégradait la situation commerciale et financière de la SNCF, notamment pour les trains régionaux.

Le fait de procéder à une expérimentation révèle une attitude intéressante, et pour tout dire nouvelle, de la puissance publique : celle de l'apprentissage. Loin de considérer qu'une loi peut à elle seule traiter un problème, la phase d'expérimentation, et la généralisation qui l'a suivie, ont inscrit les acteurs du transport régional dans un processus collectif d'apprentissage. La durée devient dès lors un facteur clé, car c'est elle qui aide à l'éclosion de nouveaux objectifs et de nouveaux comportements. Aujourd'hui, dix années après le votre de la loi SRU et près de 14 années après les premières expérimentations, nous avons le recul nécessaire pour évaluer ce processus d'apprentissage, son cheminement, ses résultats et ses perspectives.

Nous le ferons en commençant par une rétrospective. Nous verrons les régions et la SNCF, mais aussi les usagers et des élus locaux, faire ensemble le chemin qui a conduit à une sensible amélioration de l'offre TER. De ce processus collectif d'apprentissage, les formes et le contenu méritent d'être précisés. Il faut notamment distinguer ce qui relève de l'exploitation et de la mise en œuvre d'une offre ferroviaire spécifique (matériels, fiches horaires, cadencements, gares, parkings etc.) et ce qu’a été la réponse des usagers, manifeste dans l'évolution des trafics.

Dans un second temps, nous proposons un regard prospectif. Le succès même de la régionalisation invite à poursuivre le processus d'apprentissage, fut-ce en s'approchant de transformations qui relèvent sans doute plus de la discontinuité que de la continuité. Avec l'acte II de la régionalisation, pour reprendre l'expression du second rapport Haenel sur le sujet (2008), se profilent des défis majeurs autour de la question du financement, de l'ouverture à la concurrence et, finalement, dernier point mais non le moindre, des limites du subventionnement de la mobilité.

\section{Le pari "gagnant-gagnant» de l'Acte I de la régionalisation ferroviaire en France}

Dix ans après le vote de la loi SRU, qui marque l'acte I de la régionalisation ferroviaire en France, et alors que la concurrence frappe désormais à la porte de la SNCF pour le trafic intérieur de voyageurs, il nous semble possible de dresser une première évaluation ex post. Si les avis restent partagés, certains observateurs sont élogieux sur le chemin parcouru. Pour un des pères de la régionalisation ferroviaire, le sénateur $H$. Haenel (2008), « La régionalisation ferroviaire est la principale réussite de la décentralisation française ». L'analyse de la Cour des Comptes (2009) est plus réservée. Pour elle, au final, le transfert aux régions du transport express régional présente un bilan mitigé, mais des évolutions à poursuivre.

Quoiqu'il en soit, aujourd’hui, le TER est devenu un élément important de la mobilité quotidienne des Français. En 2008, le TER c'est plus de 700000 voyageurs par jour (2 millions pour le francilien, le RER), 5700 trains, plus de 800 autocars, mais aussi 2600 gares et 420

\footnotetext{
${ }^{1}$ Loi Solidarité et Renouvellement Urbains (dite loi SRU) du 13 décembre 2000, Section 5, dans ses articles 124 à 139.
} 
points d'arrêts routiers. Pour la SNCF (EPIC), le TER, qui représente 4,7 milliards d'euros de ses 20 milliards d'euros de chiffre d'affaires en 2009, est aussi le secteur qui enregistre la plus forte progression des recettes, avec $+3,5 \%$, dans un contexte de repli général de l'activité (Comptes des transports en 2009, p. 88).

Dans les lignes qui suivent, nous défendons la proposition selon laquelle, le renouveau de la mobilité de proximité, par l'intermédiaire des TER, repose avant tout sur une dynamique d'apprentissage forte, mais encore inaboutie entre l'ensemble des acteurs du système TER. Dans le cadre de la loi SRU, ces acteurs sont, les voyageurs, les régions, comme autorité organisatrice de transport (AOT), la SNCF comme exploitant unique et l'État comme bailleur de fonds. Pour étudier les conditions de ce succès et en appréhender les contours, nous distinguerons, successivement, la relation entre la SNCF et ses clients, les voyageurs du TER (1.1.); les relations entre la SNCF et ses clients institutionnels, les régions (1.2.); et enfin, le coût pour les finances publiques (1.3.).

\subsection{La relation entre la SNCF et ses clients : du désamour au renouveau}

Les statistiques sont sans appel. Le trafic régional de voyageurs connaît depuis 1997, avec l'expérimentation de la régionalisation ferroviaire, et plus encore depuis 2002, une sensible augmentation du trafic. Cette progression, continue et de plus en plus soutenue, marque une véritable rupture avec la période précédente.

Figure 1. Évolution de la fréquentation des TER depuis 1989.

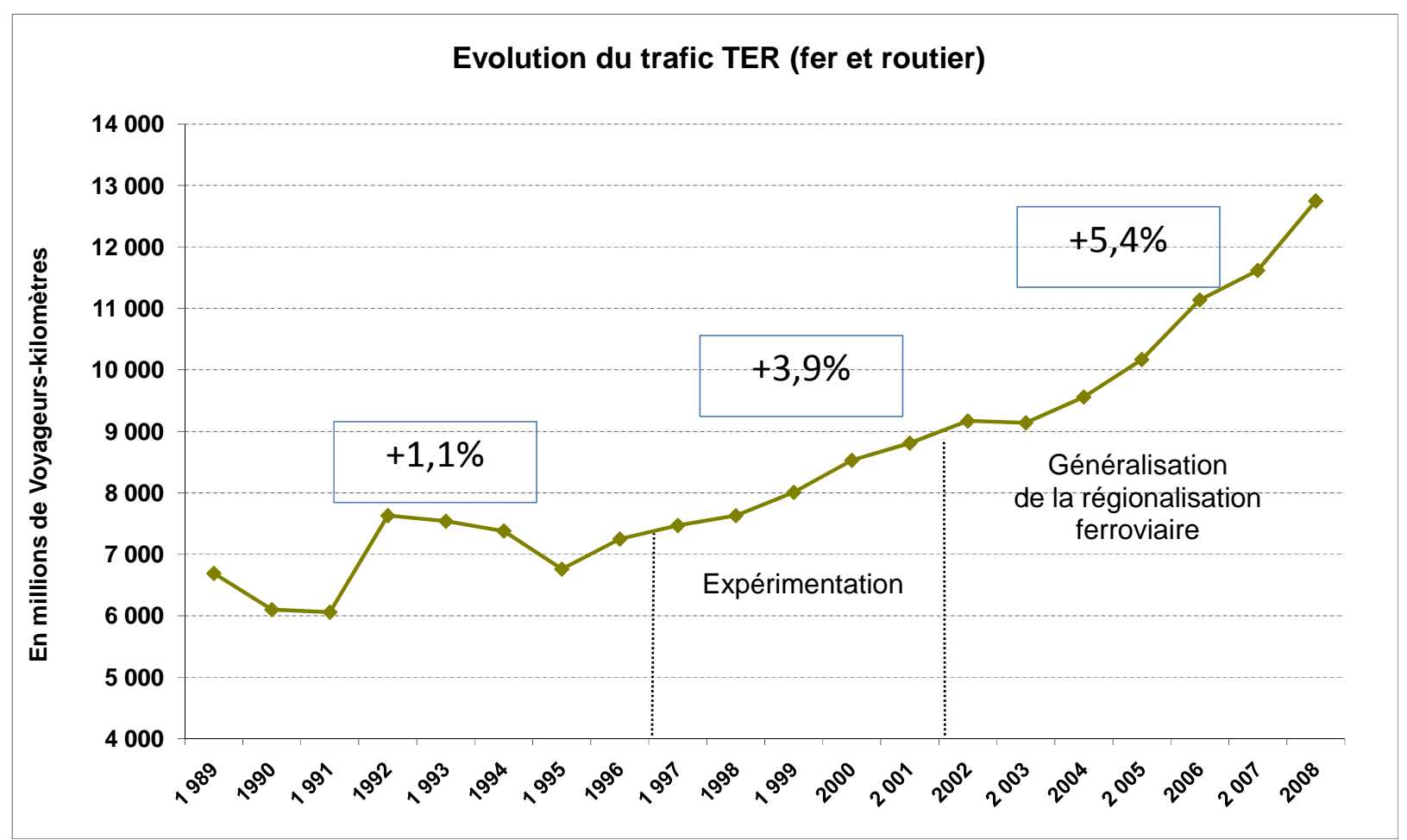

Source : Nos calculs d'après les données du Mémento de statistiques des transports, 2010.

Note : Par "routier", nous entendons les liaisons TER assurées par autocars.

Le renouveau de l'attractivité du TER est particulièrement manifeste si l'on prend un peu de recul historique. Grosso modo, alors que le trafic régional de voyageurs en France au début de la décennie quatre-vingt-dix plafonnait à environ 5600 millions de voyageurs-kilomètres par an, fin 2008, nous en sommes à 12750 millions de voyageurs-kilomètres, soit un doublement du trafic ou encore une progression de plus de 4,8\% par an en moyenne depuis le début de la 
régionalisation ferroviaire en 1997. Ce renouveau du TER s'amplifie d'ailleurs (Figure 1). Alors que le trafic TER a augmenté de 3,9\% par an en moyenne sur la période "d'expérimentation", cette hausse est de 5,4\% en moyenne depuis début 2002.

Cette véritable renaissance du transport collectif régional de voyageurs n'était pas acquise. Au début du processus, P. Zembri (1997) mentionnait combien, à partir de 1992, le TER était entré dans une crise devenue structurelle. Crise multiple, marquée par un coup d'arrêt à la hausse du trafic, par une brutale dégradation des bilans des conventions région/SNCF pesant sur les finances régionales et par une méfiance croissante entre les AO régionales et leur exploitant. Dans certaines régions, le bilan était même alarmant. Ainsi, par exemple, en PACA, le trafic régional de voyageurs avait régressé de 12,2\% entre 1989 et 1993 (de 12,5\% depuis le maximum de 1990). Dans le Limousin, la situation était plus périlleuse encore avec une baisse continue du trafic de plus de 21\% entre 1985 et 1993, c'est-à-dire depuis le début du conventionnement de cette Région avec la SNCF ! Bien rares étaient les régions qui échappaient à ce déclin général. En 1993, seules cinq régions enregistraient encore une progression du trafic dont le Nord-Pas-deCalais et l'Alsace. Circonstances aggravantes, le système de conventionnement d'alors fait porter l'intégralité de ces pertes de recettes aux régions sommées d'équilibrer les comptes, sans avoir aucunement la main ni sur les coûts, ni sur les recettes. Face à cette situation, de nombreux Conseils régionaux refusèrent de combler ces déficits conduisant l'Association des élus régionaux à demander une expertise des relations financières entre la SNCF et les régions. Cette période était aussi marquée, à la SNCF, par un état d'esprit peu favorable au TER, considéré comme technologiquement désuet (comparativement au TGV), peu performant commercialement et finalement pénalisant pour la Société nationale. Nombreux étaient alors tentés d'abandonner des dessertes et de fermer certaines lignes trop coûteuses en termes d'entretien de l'infrastructure (Campagne, 2004, p. 17).

Dans un contexte contractuel bien différent, l'expérimentation allait produire des fruits plus doux (Bonnet et alii., 2001, Faivre d'Arcier, 2002 ; CSSPF, 2002). Reposant sur une forte implication des sept régions concernées, l'expérimentation se traduisit par une amélioration qualitative et quantitative de l'offre (de 17\% environ de 1996 à 2000). Le retour de ces efforts fut rapidement au rendez-vous. La fréquentation progressa de presque $22 \%$, tout comme les recettes $(+21 \%)$.

Cette dynamique n’a pas uniquement concerné les régions expérimentatrices. Les treize autres régions, anticipant probablement sur l'extension du processus de régionalisation (CSSPF, 2002), ont également accru leur offre TER (+9\%), réussissant, elles aussi, à enclencher une hausse du trafic et des recettes (de 17\% environ).

La généralisation de la régionalisation ferroviaire, introduite par la loi SRU allait approfondir cette dynamique de succès. Les statistiques d’évolution du trafic au cours des années 2000 le confirment clairement. 
Tableau 1. Évolution du trafic et de l'attractivité du TER depuis la régionalisation.

\begin{tabular}{|l|r|r|r|r|r|r|}
\hline & \multicolumn{2}{|c|}{$\begin{array}{c}\text { Ensemble des vingt } \\
\text { régions concernées par } \\
\text { la loi SRU }\end{array}$} & \multicolumn{3}{|c|}{ Sept régions expérimentatrices } \\
\hline & $\begin{array}{c}2009 \\
\text { Indice, } \\
\text { base } 2001\end{array}$ & $\begin{array}{c}\text { TVAM (4) } \\
2001-2009\end{array}$ & $\begin{array}{c}2009 \\
\text { Indice, } \\
\text { base } 2001\end{array}$ & $\begin{array}{c}\text { TVAM (4) } \\
2001-2009\end{array}$ & $\begin{array}{c}1996-2009 \\
\text { Indice, } \\
\text { base } 1996\end{array}$ & $\begin{array}{c}\text { TVAM (4) } \\
1996-2009\end{array}$ \\
\hline $\begin{array}{l}\text { Offre kilométrique fer } \\
\text { et routier (1) }\end{array}$ & 124,2 & $+2,7 \%$ & 122,7 & $+2,6 \%$ & 146,7 & $+3,0 \%$ \\
\hline $\begin{array}{l}\text { Trafic - Voyageurs-km } \\
\text { fer et route (2) }\end{array}$ & 145,5 & $+4,8 \%$ & 145,4 & $+4,8 \%$ & 184,3 & $+4,8 \%$ \\
\hline $\begin{array}{l}\text { Ratio d'attractivité : } \\
\text { (3) = (2) / (1) }\end{array}$ & 117,1 & $+2,0 \%$ & 118,5 & $+2,1 \%$ & & 125,6 \\
\hline
\end{tabular}

Source : Nos calculs d'après les données des régions et de la SNCF.

(3) Le ratio d'attractivité est le rapport entre le trafic, exprimé en voyageurs-kilomètre (fer et routier) et l'offre de service, mesurée par le nombre de kilomètres effectués en train et bus TER.

(4) TVAM : taux de variation annuel moyen.

Le trafic TER, tous modes confondus (fer et route), a augmenté de plus de $45 \%$ entre fin 2001 et fin 2009 (Tableau 1). Cela signifie une hausse de la fréquentation de 4,8\% par an en moyenne, très supérieure à ce qu'a été dans le même temps la hausse du trafic routier. Et encore, l'année 2009, caractérisée par une très médiocre conjoncture économique, se répercutant sur les flux de transport, tend à minorer cette progression des années 2000.

Cette dynamique de la fréquentation s'est accompagnée d'une profonde réorganisation des dessertes, voire de leur cadencement, comme en Rhône-Alpes en 2008, mais également d'une densification de l'offre. Le volume d'offre est, fin 2009, supérieur de plus de $24 \%$ à son niveau d'avant la régionalisation.

Cette croissance supérieure du trafic TER par rapport à l'offre de service implique une autre performance, l'amélioration de l'attractivité de ce mode de transport. Ainsi, le taux de remplissage des TER, exprimé par le ratio des voyageurs-kilomètre rapportés au nombre de kilomètres parcourus, s'améliore, surtout depuis 2005. Avec 70 passagers par kilomètre TER (fer et route), il est en 2009 plus élevé de 17\% par rapport à fin 2001, retrouvant son niveau de 1994. Toutefois, ces évolutions moyennes ne doivent pas dissimuler une très forte variabilité du taux de remplissage selon le jour (semaine, week-end), la tranche horaire (heure de pointe ou heure creuse), le type d'axe, le sens de circulation (pointe, contre-pointe) et le mode (fer ou route).

Les résultats affichés par les sept régions expérimentatrices, depuis début 1997, début de la régionalisation, témoignent de la continuité et de la puissance des effets des processus d'apprentissage en cours : une offre accrue de presque $50 \%$, une hausse des trafics de $84 \%$ et, au final, une amélioration de l'attractivité du TER de plus d'un quart (Tableau 1).

Ceci étant, ces résultats d'ensemble mériteraient d’être affinés, région par région. Nous relèverons combien la diversité des performances entre régions est grande en matière d'augmentation de la fréquentation ou d'attractivité de leur réseau TER.

Ainsi, parmi les sept régions dites expérimentatrices, l’Alsace, Rhône-Alpes et les Pays de la Loire ont enregistré les plus fortes progressions de trafic, supérieures à la moyenne. Si la 
progression de la région PACA est sensiblement équivalente à la moyenne, celle du Nord-Pasde-Calais, de la région Centre et du Limousin, sont moins dynamiques, bien que toutes positives, ce qui, dans le cas du Limousin, n’était pas acquis (Figure 2).

Figure 2. Évolution de la fréquentation TER des régions expérimentatrices.

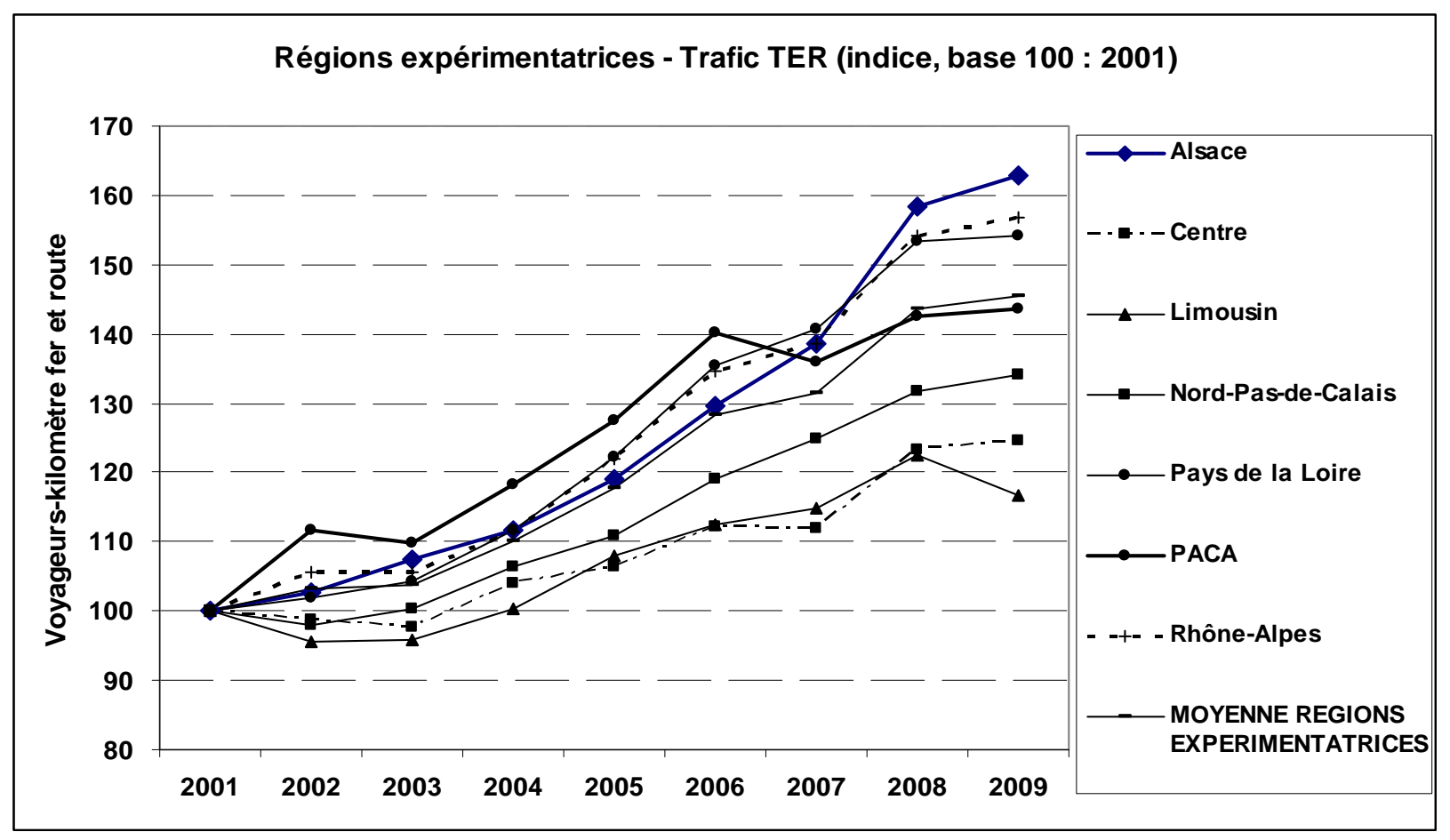

Source : Nos calculs d'après les données des régions et de la SNCF.

Concernant les taux de remplissage, si le TER améliore ses performances dans toutes les régions, de sensibles disparités demeurent. Sans surprise, les régions les plus urbaines connaissent les taux de remplissage les plus élevés, telles PACA avec 89 voyageurs par kilomètre en 2008 (l'année 2009 fait exception) et le Nord-Pas-de-Calais avec 86, et inversement pour des régions rurales, telles le Limousin avec, en moyenne, moins de 30 voyageurs par kilomètre TER. Nous noterons que le volontarisme régional en matière d'offre TER n'est pas nécessairement la clé du succès du taux de remplissage : ainsi, alors que le Nord-Pas-de-Calais a augmenté son offre de $25 \%$ depuis 2001, l'attractivité moyenne de ses TER s'est accrue de seulement 7\% environ. Dans le même temps, le Limousin qui n'a augmenté son offre que de 2,5\%, voit l'attractivité de ses TER s'accroître de presque $14 \%$. 
Tableau 2. L'évolution de l’attractivité des TER avec la régionalisation.

\begin{tabular}{|c|c|c|c|c|c|c|c|c|c|c|}
\hline & 2001 & 2002 & 2003 & 2004 & 2005 & 2006 & 2007 & 2008 & 2009 & $\begin{array}{l}2009, \text { base } \\
100: 2001\end{array}$ \\
\hline Alsace & 61 & 61 & 62 & 63 & 67 & 72 & 71 & 78 & 78 & 127,6 \\
\hline Centre & 66 & 64 & 63 & 66 & 68 & 74 & 73 & 79 & 79 & 119,8 \\
\hline Limousin & 25 & 24 & 24 & 24 & 25 & 26 & 26 & 28 & 28 & 113,9 \\
\hline $\begin{array}{l}\text { Nord-Pas-de- } \\
\text { Calais }\end{array}$ & 80 & 78 & 76 & 74 & 73 & 78 & 79 & 86 & 86 & 107,3 \\
\hline Pays de la Loire & 62 & 62 & 62 & 63 & 67 & 75 & 78 & 85 & 85 & 137,1 \\
\hline$P A C A$ & 77 & 76 & 72 & 76 & 79 & 87 & 79 & 89 & 79 & 102,0 \\
\hline Rhône-Alpes & 63 & 63 & 62 & 64 & 70 & 74 & 75 & 77 & 77 & 121,6 \\
\hline $\begin{array}{l}\text { Régions } \\
\text { expérimentatrices }\end{array}$ & 65,4 & 64,7 & 63,7 & 65,2 & 68,5 & 73,7 & 73,4 & 78,4 & 77,5 & 118,5 \\
\hline
\end{tabular}

Source : Nos calculs d'après les données des régions et de la SNCF.

Les clés de ces succès sont davantage à rechercher dans la qualité de la contractualisation et plus encore dans les dynamiques d'apprentissage à l'œuvre entre les deux principaux acteurs, l'exploitant, la SNCF et les AOT, les régions.

Si le pari de la régionalisation ferroviaire en France était de répondre à la sévère crise d'attractivité du trafic régional de voyageurs, tout en préservant la situation de monopole de la SNCF, dans l'espoir de lui permettre d'accroitre sa capacité d'adaptation face à des marchés du transport de plus en plus concurrentiels, ce double pari semble globalement gagné.

Alors que les voyageurs du TER sont les clients de la SNCF, le conventionnement de ce service public de transport de voyageurs fait des Conseils régionaux, les « clients institutionnels » de l'opérateur historique. Pour cette raison, il nous importe maintenant de présenter les contours de cette contractualisation.

\subsection{La contractualisation SNCF-régions : du « partenariat obligé » à une dynamique de coopération}

Le partenariat entre les Conseils régionaux et la SNCF n'est pas nouveau. L'histoire de cette relation, que nombre d'observateurs considèrent comme mouvementée (Zembri, 1997; Campagne, 2004 ; Barone, 2008), mais désormais fructueuse, est marquée par trois étapes principales : 1982, 1997 et 2002.

Les lois de décentralisation de 1982 d'abord. Ainsi, la LOTI ${ }^{2}$ retient le principe de transfert de la compétence transport aux différents niveaux de collectivité et assigne aux régions le double rôle d'organisateur du transport à l'échelle de leur territoire et de gestionnaire de l'intermodalité, mais sans rendre cette compétence obligataire et, surtout, sans établir précisément les modalités pratiques de ce transfert et de ce conventionnement. En conséquence, les régions, sans réel transfert de compétences et de moyens, ne seront impliquées qu'à la marge dans les modifications d'offre, tout en ayant à supporter de fortes hausses de leur contribution.

\footnotetext{
${ }^{2}$ La loi 82-1153 du 30 décembre 1982, d'orientation des transports intérieurs (LOTI), fixe le cadre général de la politique des transports en France.
} 
Face à cette impasse, le rapport Haenel (1993) recommanda d'envisager une expérimentation du principe de régionalisation ferroviaire ${ }^{3}$. Cette dernière, qui fit des régions des AOT à part entière, s'appuya sur un transfert réel de compétences et de moyens, et positionna la SNCF comme prestataire unique, sommé d'améliorer ses coûts et de développer une politique commerciale dynamique.

En 2000, suite au bilan positif de l'expérimentation, le législateur décida, avec la loi SRU, de faire de toutes les régions métropolitaines (sauf la Corse et l'Ile de France) des AOT de plein exercice. Comment ont évolué depuis les relations entre les régions et la SNCF ?

Nous soutiendrons que ces relations ont été sujettes à un intense phénomène d'apprentissage, marqué par des tâtonnements et surtout par un rééquilibrage en faveur des régions. Pour illustrer cette dynamique, nous reprendrons le propos du Directeur du conventionnement TER à la SNCF, lors de la signature des conventions de 2002.

La régionalisation a permis à la SNCF de développer une nouvelle culture de proximité régionale et locale. Les régions se sont dotées de services compétents et de plus en plus étoffés. La SNCF a découvert puis professionnalisé son métier de délégataire, répondant à une autorité publique régionale.

Un nouvel équilibre s'installe entre les autorités organisatrices et la SNCF ; les relations entre autorité et exploitant, après une première phase de découverte et de mise au point, s'installent, C. Steinmetz, 2005, p. 55.

Pour approfondir ces mutations qui ont permis à la SNCF de passer, aux yeux des régions, d'un statut de prestataire unique et redouté à celui d'un partenaire actif inscrit dans une relation plus équilibrée et mutuellement avantageuse, nous distinguerons d'abord les adaptations qui ont affectées la SNCF, puis celles concernant les régions.

La SNCF, pour répondre aux exigences de ses nouveaux clients institutionnels, les Conseils régionaux, a dû se plier à une réorganisation industrielle profonde et souscrire à une véritable révolution culturelle. La mise en place d'une nouvelle organisation industrielle s'est d'abord concrétisée par la création des DTER, les Directeurs régionaux TER. Ces derniers assument un double rôle, celui de vis-à-vis des régions dans le suivi de la contractualisation et celui d'interface avec tous les services internes de la SNCF. La mise en place d'un "pilotage par axe », sous la responsabilité de "managers de ligne», est ensuite venue progressivement compléter cette prise en compte des acteurs du territoire. Sous l'impulsion de certaines régions, des instances d'expression des clients, des élus et des usagers sont venues compléter le dispositif, sous le nom de « Comité de ligne » ou de « Comité de desserte ».

La SNCF s'est également efforcée de mettre en place une véritable politique de gestion de la relation avec ses clients du TER. L'objectif était double : fidéliser la clientèle existante et conquérir de nouveaux publics. A cette fin, les conventions TER ont fréquemment introduit un système d'intéressement aux recettes, ainsi que des bonus-malus pour sanctionner financièrement l'opérateur au regard de la qualité du service. Mais ces dispositifs d'incitation n'ont pas réussi à surmonter tous les problèmes. La qualité produite est régulièrement inférieure aux objectifs fixés en matière de ponctualité et de continuité du service. Cet état de fait amène la SNCF à être redevable de pénalités conséquentes qui minore substantiellement, parfois, la rentabilité de son activité TER. Face à cette insuffisance récurrente de qualité produite, les usagers du TER tendent

\footnotetext{
${ }^{3}$ La régionalisation ferroviaire vient s'inscrire dans la réforme ferroviaire de 1997 qui allait aussi donner naissance à RFF en vertu de la loi 97-135 du 13 février 1997.
} 
désormais à se mobiliser et à faire valoir leurs droits, en interpellant tant la SNCF que les régions, pour les mettre face à leur responsabilité ${ }^{4}$.

Au total, la régionalisation ferroviaire a été l'occasion pour la SNCF de vivre une sorte de révolution culturelle, qui lui a imposé d'appréhender le transport régional, non plus d'abord en fonction de contraintes techniques de production, mais selon les besoins exprimés par les acteurs des territoires. Nombre d'élus n’ont pas hésité à rendre hommage de ces mutations des cheminots eux-mêmes qui ont accepté de «jouer le jeu » et de remettre en cause un grand nombre d'habitudes (H. Haenel, 2008, p. 10).

Les régions, elles aussi, ont dû s’adapter pour intégrer cette responsabilité qui, pour la première fois, les confronte à la gestion d'une compétence présentant une forte composante technique. Le pilotage effectif de la politique TER supposait que les AOT régionales acquièrent les capacités d'expertise qui leur faisaient initialement défaut (Bonnet et alii., 2001). Cette prise en main s'est traduite par une intense politique de recrutement, mais aussi par le recours massif à des expertises externes assurées tant par des cabinets conseils, des bureaux d'études ou des sociétés d'ingénierie (A. Faure, 2007). Si les services transport des conseils régionaux se sont partout étoffés, ils restent très généralement notoirement inférieurs à ceux des activités TER de la SNCF. Dans certains cas cependant, la région a su inverser l'asymétrie d'information initiale, comme cela semble être le cas en Rhône-Alpes par exemple (A. Faure, 2007).

Un deuxième argument vient alimenter la thèse selon laquelle les régions ont souhaité, et réussi d'emblée, à inscrire leur empreinte sur le cours de la régionalisation ferroviaire, l'écriture même des conventions TER cosignées par la SNCF. C. Desmaris (2004) avait montré que les conventions de 2002 sont très hétérogènes d’une région à une autre, manifestant une réelle liberté dans l'écriture de leur contrat avec leur vis-à-vis obligé, la SNCF. Nous avions également identifié l'émergence de phénomènes d'apprentissage, qui se traduisent par un approfondissement contractuel marqué par une certaine complexification des mécanismes et par une relative standardisation des procédures. Ces deux caractéristiques témoigneraient, selon l'auteur, d'une progression de la rationalité bureaucratique-légale, au sens wébérien, dans la contractualisation des régions avec leurs partenaires.

Nous observons enfin combien les régions se sont diversement, mais néanmoins sensiblement, investies dans le développement de l’offre de transport de voyageurs. Elles ont généralement rationnalisé les dessertes, pour les adapter aux particularités de leur territoire, tout en augmentant la densité du service offert. Elles ont aussi visé à améliorer l'attractivité du TER, en renouvelant la majorité du matériel roulant, au prix d’une prise en charge financière conséquente, mais aussi, de plus en plus fréquemment, en acceptant de cofinancer la modernisation de segments d'infrastructure et de gares.

Au-delà de cette montée en puissance de l'expertise régionale et de la prise de pouvoir de ces AO sur cette compétence nouvelle, un certain nombre de difficultés subsistent. L’opacité des méthodes d'établissement des comptes de la SNCF, méthodes sans cesse modifiées unilatéralement, est encore très souvent décriée. La mise en place d'une véritable comptabilité par ligne, ardemment souhaitée par les régions, n’a pas encore véritablement été déployée ${ }^{5}$. La

\footnotetext{
${ }^{4}$ Ainsi en 2010 et 2011, des usagers, réunis en associations, protestant contre des dysfonctionnements à répétition sur leurs lignes, ont exigé de la SNCF des indemnisations compensatrices et ont lancé des mots d'ordre appelant au boycott des contrôles des billets. Certains Présidents de région, tels celui de Rhône-Alpes, donnant écho à ces mécontentements, ont également sommé la SNCF de s'expliquer sur ces dysfonctionnements en série et ont exigé la mise en œuvre de mesures fortes et rapides à même de les résoudre. Face à cette exaspération, le 20 janvier 2011, la Direction de la SNCF a dû annoncer un plan pour renforcer la qualité de ses services sur les lignes les plus atteintes dont la mise en œuvre se déroulera sur deux ans. Reste à savoir si ce plan, qui vise principalement à réaliser d'importants travaux de remise à niveau de l'infrastructure, suffira.

${ }^{5}$ Notons que certaines Régions, lors de la signature des conventions de deuxième génération, ont exigé la création de comptes de lignes. C’est le cas de Rhône-Alpes, du Nord-Pas-de-Calais et de la Bourgogne.
} 
Cour des comptes (2009, pp. 35-38), elle-même, insiste sur cette carence. D’autres sujets de discordes apparaissent ponctuellement, notamment la question du partage des responsabilités en matière de communication ou celui du statut des services en gares. Mais globalement le rééquilibrage est en cours et l'asymétrie d'information initiale en partie comblée.

\subsection{Le coût pour les finances publiques: considérable, mais encore soutenable}

Qui paye le TER ? Si toutes les parties s'accordent pour estimer que le TER coûte à la Collectivité, tant à l'État, qui initialement s'était engagé à transférer cette compétence aux régions en respectant le principe de neutralité financière, qu'aux régions qui se sont largement impliquées pour améliorer l'offre, la tarification et le matériel roulant, une incertitude demeure sur le montant exact du coût complet du TER et sur sa répartition entre les acteurs.

\subsubsection{Une régionalisation accompagnée par un effort financier considérable des Collectivités publiques}

La Cour des Comptes (2009), récapitulant les rares études disponibles, estimait, le coût complet du TER entre 4 et 5 milliards d'euros pour 2009. L'étude de la direction générale des infrastructures, des transports et de la mer (DGITM) estimait pour 2005 le coût complet à 4 milliards d'euros se répartissant en trois blocs de charges : les services fournis par la SNCF, pour 2,1 milliards d'euros, l'infrastructure avec un coût complet de 1,3 milliard d'euros (les péages versés étant de 470 millions d'euros, laissant un déficit à RFF de 878 millions d'euros), et le financement du matériel roulant à 0,6 milliard d'euros. Notons que les voyageurs ne participent que de façon résiduelle au financement du TER, dont l'essentiel du coût est porté par les contribuables. La Cour des comptes (2009) relevait, qu'en 2005, les voyageurs ne couvrent, en moyenne, que $17 \%$ environ du coût complet du TER, alors qu'ils bénéficient d'une sensible amélioration de l'offre tant quantitative que qualitative. Concernant les seules charges d'exploitation, la participation des usagers serait de l'ordre de moins d'un tiers, avec des différences notoires entre les régions.

Le transport régional de voyageurs demeure donc une activité de service public très largement subventionnée et donc dépendante des choix et des possibilités financières des Collectivités publiques. Comment le coût du TER pour les finances publiques a-t-il évolué depuis le début de la régionalisation ? En dépit des incertitudes de chiffrages de ce coût global, nous en proposerons une estimation à partir de deux sources: les rapports de la Commission des comptes des Transports de la Nation d'abord et les comptes administratifs des régions ensuite.

A suivre les données des Comptes des Transports de la Nation, l'engagement financier des régions est considérable : 3,3 milliards d'euros versés à la SNCF en 2009 et une progression de $68 \%$ au cours des huit premières années de la régionalisation ferroviaire (Tableau 3). 
Tableau 3. Les versements des régions à la SNCF pour l'activité TER.

\begin{tabular}{|c|r|r|r|r|r|}
\hline En millions d'euros courants & \multicolumn{1}{|c|}{2002} & \multicolumn{1}{|c|}{2007} & \multicolumn{1}{c|}{2008} & 2009 & $\begin{array}{c}\text { Variation } \\
\text { 2002/2009 } \\
\text { en } \%\end{array}$ \\
\hline Contribution d'exploitation & $\mathbf{1 4 0 5}$ & $\mathbf{2} \mathbf{0 5 6}$ & $\mathbf{2 2 2 4}$ & $\mathbf{2 3 2 5}$ & $\mathbf{+ 6 5 , 5 \%}$ \\
\hline Exploitation des services & 1204 & 1744 & 1906 & 1991 & $+65,4 \%$ \\
\hline Compensations des tarifs sociaux & 200 & 312 & 318 & 334 & $+\mathbf{+ 6 7 , 0 \%}$ \\
\hline $\begin{array}{l}\text { Subventions d'investissement } \\
\text { (matériel roulant) }\end{array}$ & $\mathbf{5 6 8}$ & $\mathbf{8 8 2}$ & $\mathbf{1 0 2 6}$ & $\mathbf{9 9 1}$ & $\mathbf{+ 7 4 , 5 \%}$ \\
\hline $\begin{array}{l}\text { Total des versements des régions à } \\
\text { la SNCF au titre du TER }\end{array}$ & $\mathbf{1 9 7 3}$ & $\mathbf{2 9 3 8}$ & $\mathbf{3 2 5 0}$ & $\mathbf{3} \mathbf{3 1 6}$ & $\mathbf{+ 6 8 , 1 \%}$ \\
\hline
\end{tabular}

Source : Nos calculs d'après Les Comptes des Transports de la Nation 2010 (2009, p. 117).

Si la subvention d'équilibre à l'exploitation constitue l'essentiel de la contribution régionale, avec environ $60 \%$ du total des versements à la SNCF, l'effort des régions dans la mise en place de tarifications incitatives, et plus encore leur volonté de disposer d'un parc de matériel véritablement attractif impacte fortement l'évolution du volume de leur contribution financière. Ainsi, alors que les subventions d'investissement au titre du matériel roulant ne représentaient que 570 millions d'euros en 2002, ce poste absorbe presque 1 milliard d'euros en 2009, soit 30\% des dépenses des régions au titre du transport régional de voyageurs, 75\% de plus qu'en 2002 (Tableau 3). Ces dépenses d'investissement devraient prochainement plafonner du fait de l'achèvement du renouvellement du parc. Mais, l'effort financier des régions à destination de la modernisation des gares et de l'infrastructure, viendrait prendre le relais.

Soulignons que ces versements des régions au titre du TER ne constituent pas l'effort financier réel de ces collectivités territoriales, puisque. En effet, l'État vient en compenser une partie, comme la loi de décentralisation l'avait explicitement prévue. En outre, l'effort financier des régions en faveur du TER ne comprend pas seulement les versements à la SNCF, mais aussi leurs engagements au titre de la modernisation des gares et de l'infrastructure ferroviaire qui ne sont pas pris en compte dans les Comptes transports de la nation. C'est pourquoi nous compléterons cette première approche en sollicitant les Comptes administratifs des régions.

Bien que la perspective offerte par les Comptes administratifs des régions soit plus globale que la précédente, les tendances restent les mêmes. La régionalisation de cette compétence s'est accompagnée d'une sensible progression des transferts financiers transitant par le budget des régions. Les contributions régionales sont passées d'un peu plus de 2 milliards d'euros en 2002 à 3,6 milliards d'euros en 2008 et presque 4 milliards d'euros en 2009 (Figure 3). 1,9 milliard d'euros de plus en sept ans, soit une hausse du budget total affecté au TER de $93 \%$ ! L’investissement est le poste qui augmente le plus (172\%), initialement en raison de la modernisation du matériel roulant et, depuis peu, du fait des cofinancements régionaux en faveur des gares et de l'infrastructure ${ }^{6}$.

\footnotetext{
${ }^{6}$ En 2008, les dépenses d'investissement des Régions en matériel roulant se sont élevées à 873 millions d'euros et à 449 millions d'euros pour la modernisation des gares et de l'infrastructure.
} 
Figure 3. Évolution des dépenses des régions au titre du transport régional de voyageurs.

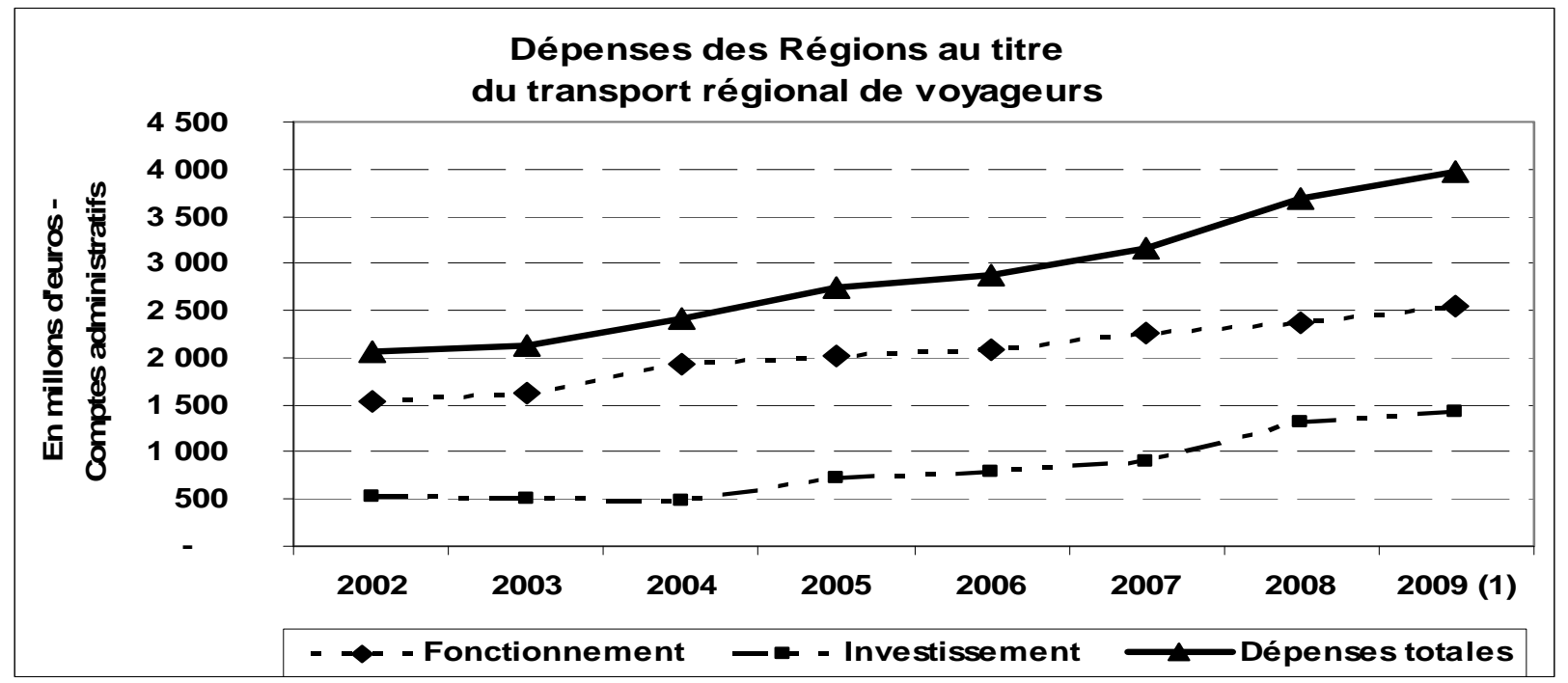

(1) Pour 2009, prévisions à partir des budgets primitifs des régions.

Source : Nos calculs d'après Les Comptes des régions, DGCL (2010).

Au total, au-delà de disparités régionales fortes, le TER représente en généralement le deuxième poste du budget des régions, avec $23 \%$ en moyenne (après l'enseignement, mais avant la formation professionnelle et l'apprentissage).

\subsubsection{Un doute sur les rendements d'échelle croissant du TER}

Une question taraude l'économiste du secteur ferroviaire. Quelle est l'importance des économies d'échelle et donc quelle est la zone de pertinence du monopole d'exploitation ? Pour J. Levêque (2007), les rendements d'échelle ne sont pas suffisamment croissants sur les réseaux régionaux en France pour justifier le maintien du monopole d'un opérateur sur chacun de ces réseaux régionaux. Dans le contexte d'ouverture à la concurrence, l'auteur recommandait aux régions d'allotir progressivement leur réseau. Sollicitant une méthodologie différente, les comptes de surplus, et avec des données plus récentes portant sur la période 2002-2007, C. Desmaris (2010b), aboutit à des résultats plus contrastés. Mais il n'en demeure pas moins que la régionalisation s'est accompagnée d'une élévation du coût unitaire de ce service public pour la Collectivité, que la mesure soit effectuée sur la base des voyageurs-kilomètres ou, plus encore, des trains-kilomètres (Figure 4 et 5). De ce point de vue, le processus d'apprentissage ne peut être considéré comme abouti.

Entre fin 2002 et fin 2008, le coût global réel (exploitation et investissement) en subvention publique du train-kilomètre a augmenté de 38,6\%, passant de 13,3 euros à 18,4 euros (Figure 4). Si l'on exclut l'effort des collectivités en matière d'investissement, où un rattrapage important était à réaliser, et pour mieux se concentrer sur l'efficience des fonds publics, nous ne rapporterons que les seules contributions d'exploitation au nombre de trains-kilomètres réalisés. La hausse est ici de 36,5\%, du même ordre de grandeur. Le coût réel en subvention publique de l'exploitation TER est passé de 9,4 euros par train-kilomètre en 2002, à 13,3 euros en 2007. Nous relèverons également qu'un tiers de cette augmentation du coût d'exploitation TER par trainkilomètre s'est effectuée au cours de la seule année 2004, marquée par une augmentation sensible des péages RFF. 
Figure 4. Évolution du coût du train-kilomètre en subvention publique (euros constants).

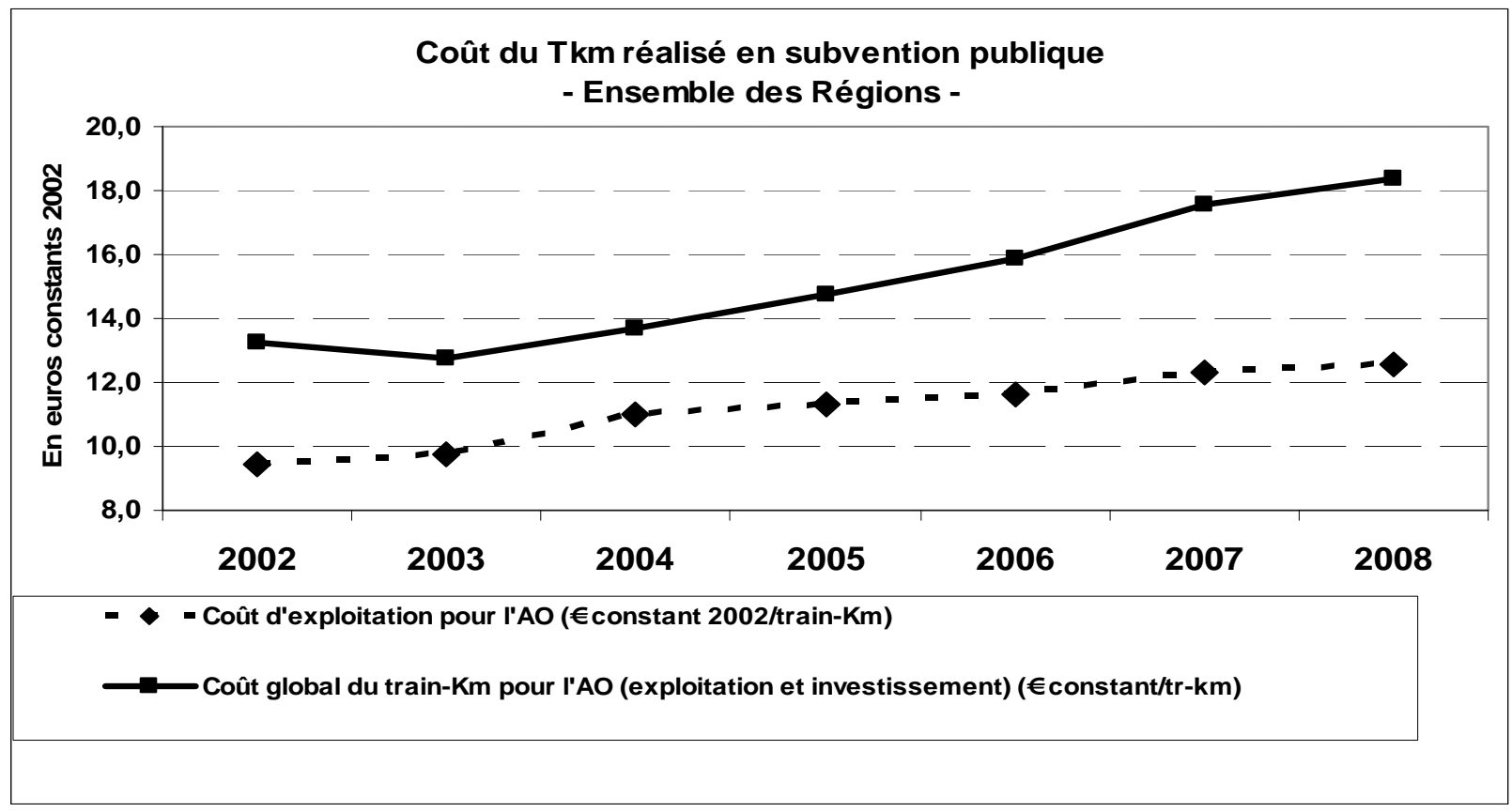

Source : Nos calculs d'après Les Comptes des régions, DGCL (2010).

Une autre perspective de l'efficience des concours publics est possible, celle qui les compare non à la production réalisée, mais au service rendu à la Collectivité, apprécié par la fréquentation du service mis à disposition du public. La variable d'étude est alors non plus le train-kilomètre, mais le voyageur- kilomètre ${ }^{7}$ (Figure 5 ).

Figure 5. Évolution du coût du voyageur-kilomètre en subvention publique (euros constants).

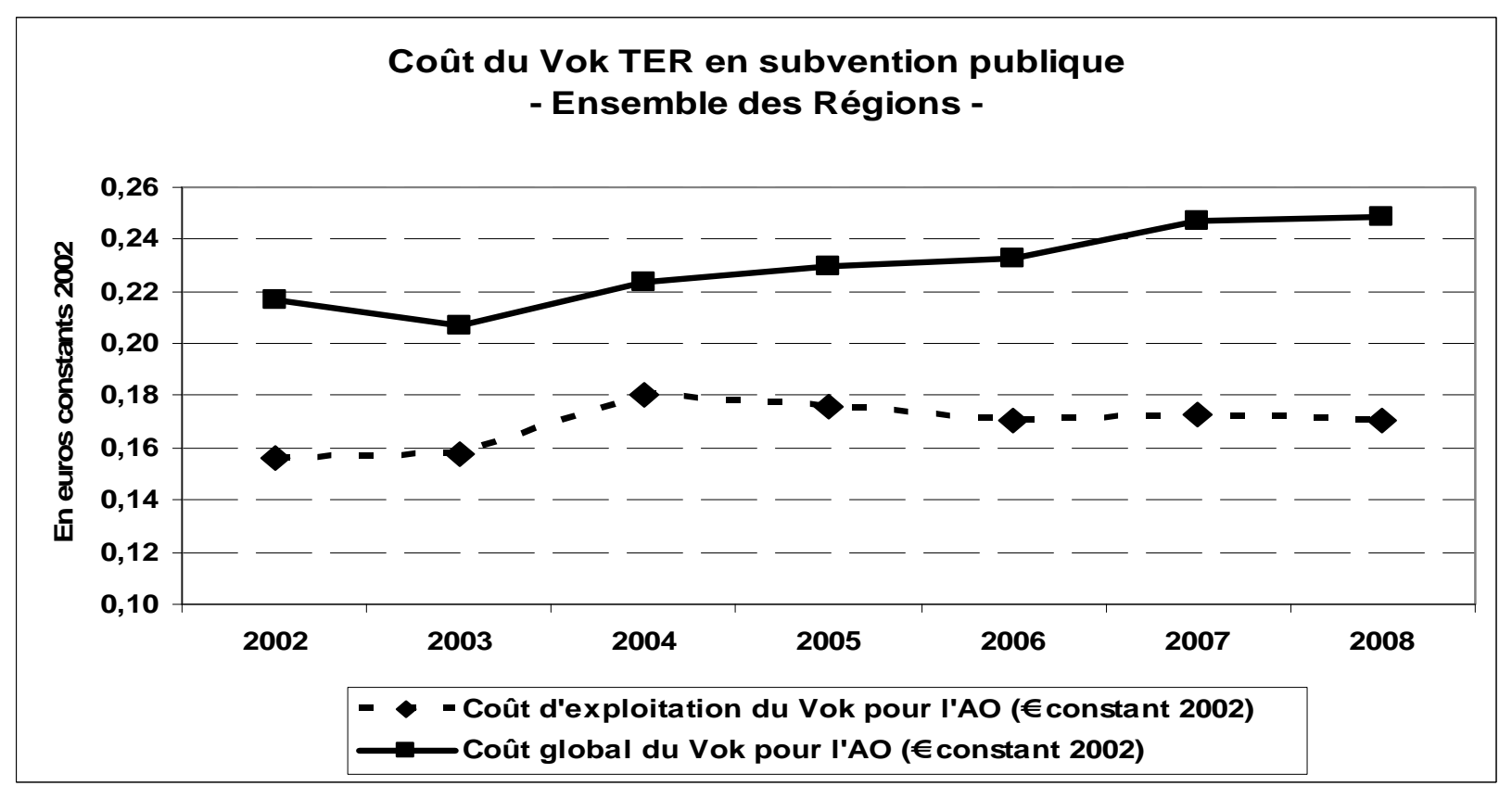

Source : Nos calculs d'après Les Comptes des régions, DGCL (2010).

\footnotetext{
${ }^{7}$ Rappelons avec insistance que pendant une brève période (1978-1981), l’État avait réussi à imposer à la SNCF un système de contribution publique bien plus incitatif que celui qui prévaut aujourd'hui. Il était fondé sur une contribution au voyageur-kilomètre, et non sur le différentiel dépenses-recettes constatés ex-post (P.-Y. CAMPAGNE, 2004, p. 16).
} 
Entre fin 2002 et 2008, le coût total du TER par voyageur-kilomètre est passé de 0,217 à 0,249 euros 2002, soit une hausse de $14,8 \%$ en valeur constante (Figure 5). Nous remarquerons que ce coût est globalement resté stable depuis 2007. Si l'on se concentre sur le seul coût d'exploitation, la hausse cumulée n'est plus que de $9 \%$ en valeur réelle, mais surtout elle se concentre sur l'année 2004. Ce coût connaît ensuite une diminution quasi-continue. L'effet de la hausse des péages vient donc fortement dissimuler un résultat inattendu, la stabilité du coût réel du voyageur-kilomètre en subvention publique.

Approfondie sur l'échantillon des sept régions expérimentatrices, une analyse des comptes SNCF-TER montre qu'il importe de distinguer les différentes composantes du coût TER facturé par la SNCF aux régions. Si les péages ont partout largement augmenté, impactant fortement le coût unitaire d'exploitation, et donc la subvention régionale d'équilibre, il n'en va pas de même pour tous les autres coûts d'exploitation. Contrairement à l'hypothèse, suggérée par la théorie standard, qui voudrait que les régions aient été victimes de l'asymétrie informationnelle en faveur de la SNCF, on ne voit pas apparaître clairement une capture de rente nouvelle par l'opérateur historique. Au contraire, la frontière de prix entre ces deux acteurs s'est plutôt déplacée en faveur des régions. Le prix des charges d'exploitation facturées par la SNCF aux régions a généralement diminué, en valeur constante, en tout cas sur notre échantillon (Figure 6). Des gains de productivité globale des facteurs ont été enregistrés, mais leur mesure reste très conventionnelle du fait de la forfaitisation des charges SNCF et, pour l'essentiel, ils demeurent largement tributaires de la dynamique d'augmentation du trafic.

Figure 6. Évolution du prix des charges d'exploitation SNCF-C1 au train-kilomètre réalisé.

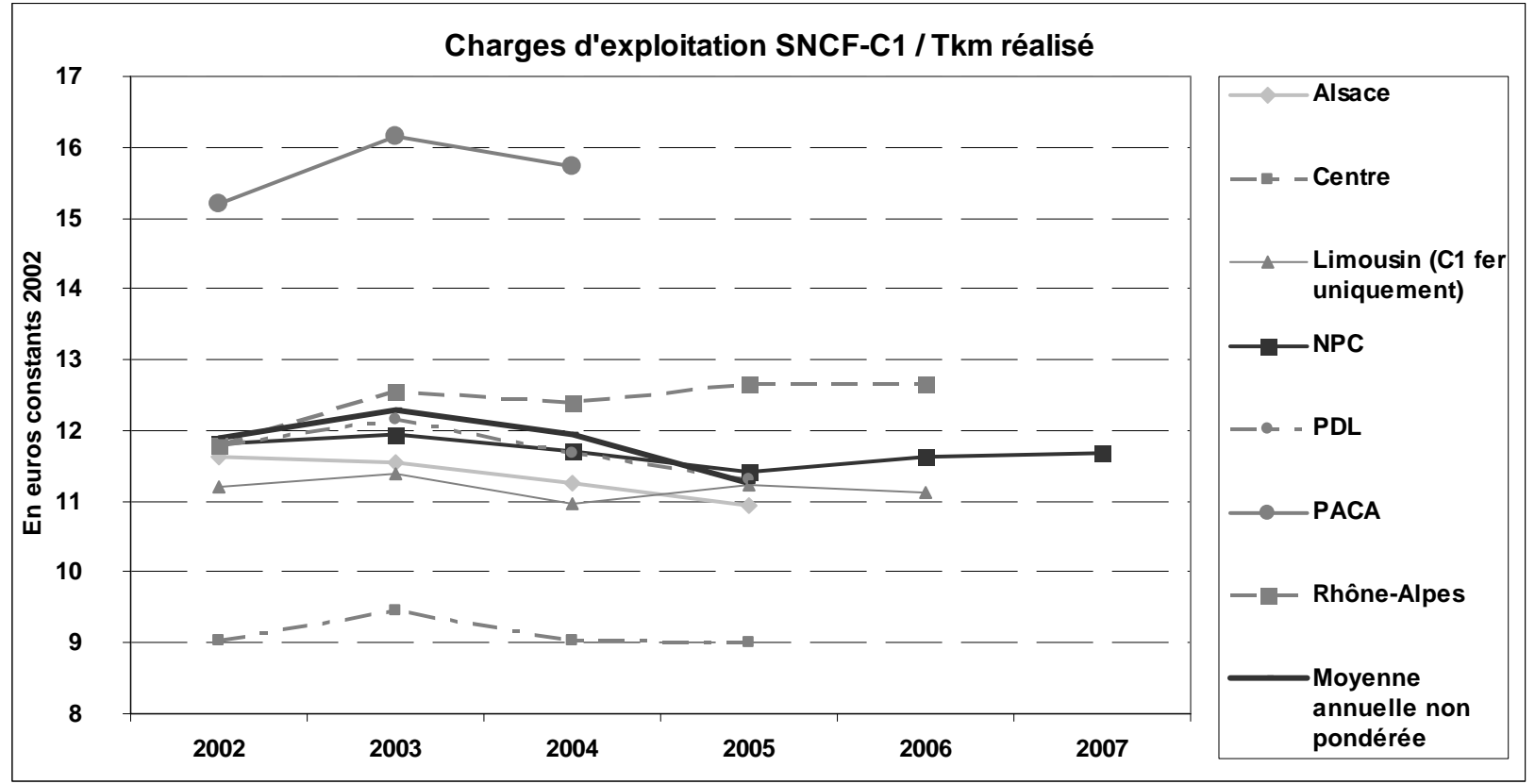

Source : Source : Nos calculs d'après Les Comptes des régions, DGCL (2010).

Quant aux régions, le bilan est plus nuancé qu’il y parait en première approche. Appréciée globalement, la contribution d'exploitation nette ${ }^{8}$ (hors subventions tarifaires et subventions de modernisation du matériel roulant), augmente, ne faisant pas vraiment apparaitre d'incidence positive d'éventuelles économies d'échelle sur la subvention régionale d'équilibre. Ceci étant,

\footnotetext{
${ }^{8}$ La contribution régionale d'équilibre aux services TER est calculée nette, c'est à dire une fois prise en compte l'incidence des éventuelles pénalités et des bonus-malus qui encadrent la qualité de la réalisation du service par l'exploitant.
} 
après une phase de hausse rapide, presque partout, s'observe un ralentissement, voire un faible mouvement de baisse, sans jamais pour autant revenir au niveau initial (Figure 7).

Figure 7. Évolution de la subvention d'exploitation nette par train-kilomètre en euros constants 2002.

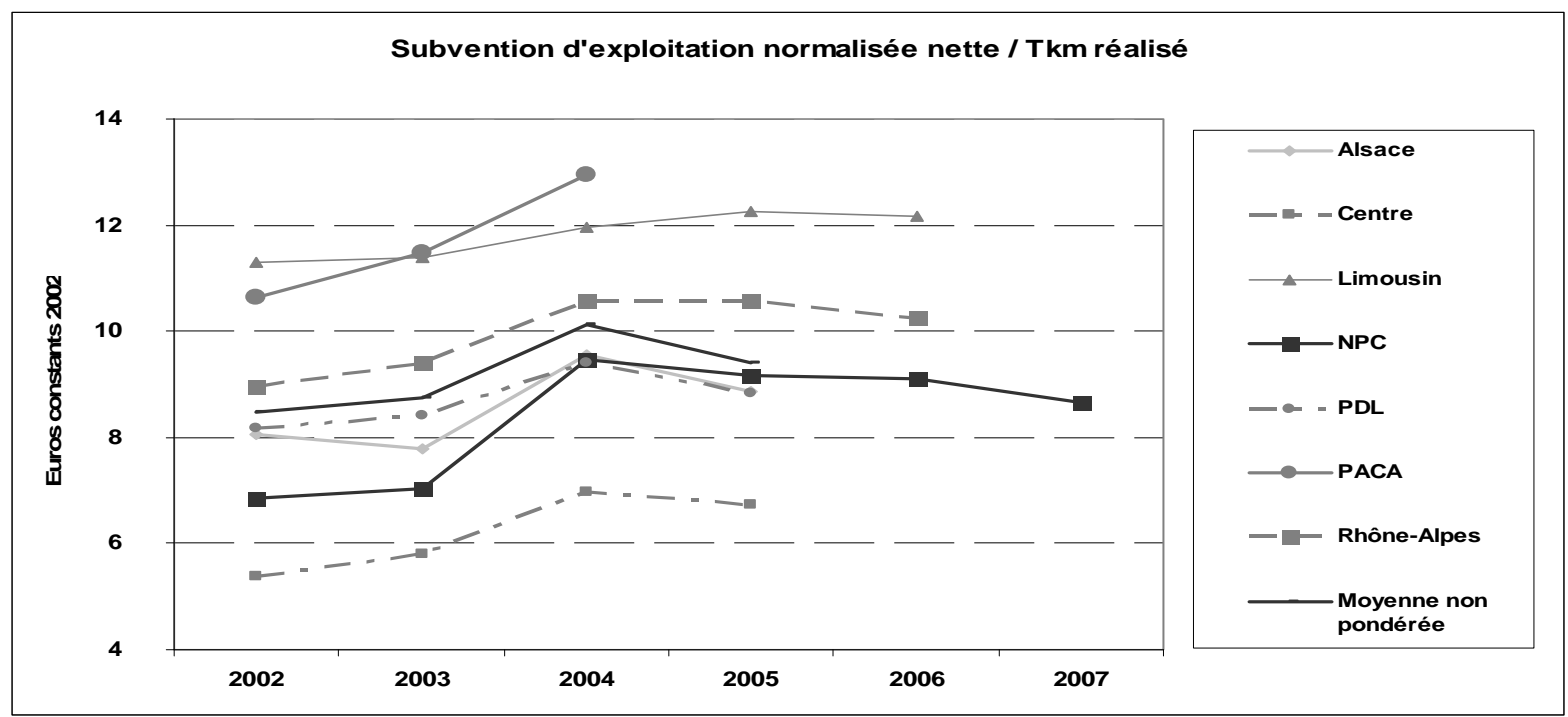

Source : Source : Nos calculs d'après Les Comptes des régions, DGCL.

La perspective, observée hors péages, est fort différente. Après une hausse, la subvention d'exploitation des régions est en moyenne stable en fin de période, avec une baisse dans plusieurs des sept régions de notre échantillon, notamment l'Alsace et les Pays de la Loire (Figure 8).

Figure 8. Évolution de la subvention d'exploitation nette, hors-péages, par train-kilomètre.

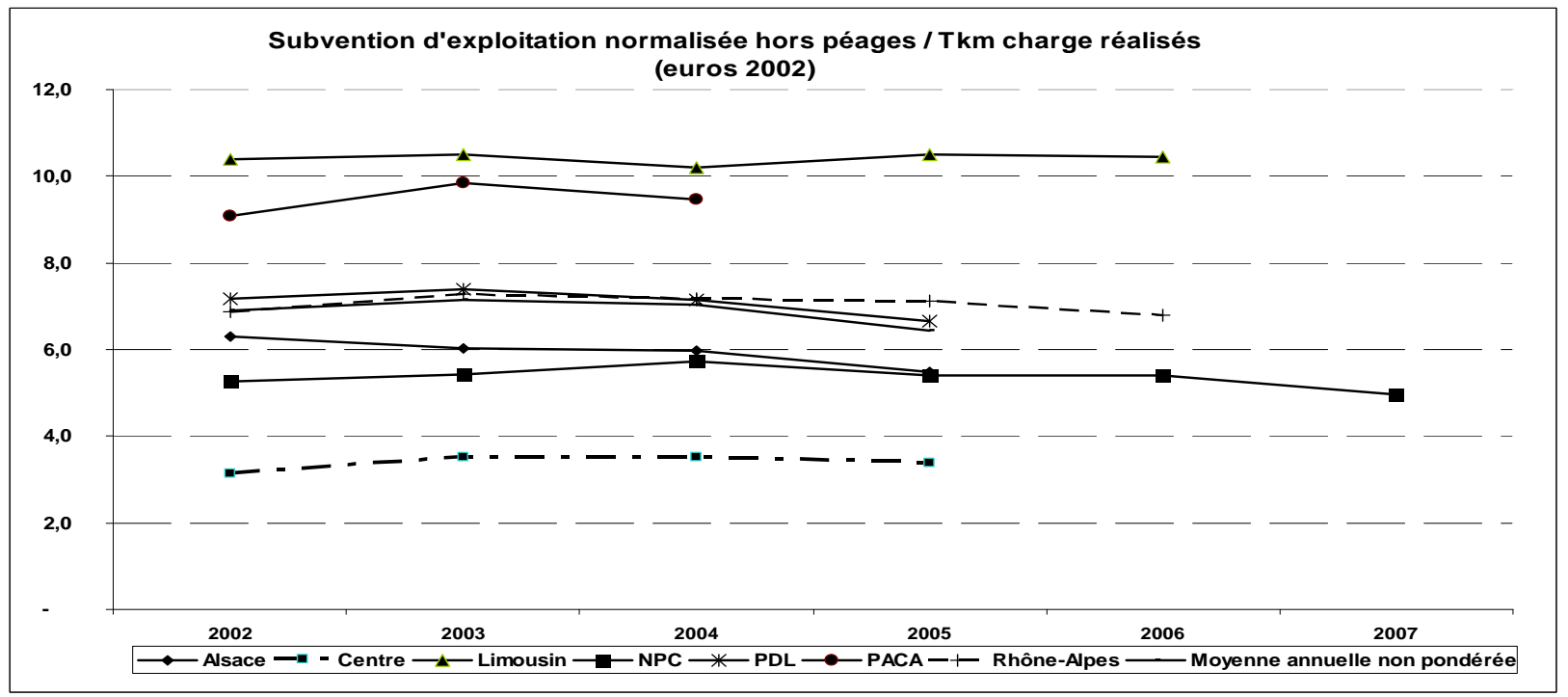

Source : Nos calculs d'après Les Comptes des régions, DGCL.

Ce résultat, inattendu, est tout à fait appréciable. Il signifie que les régions, après une période d'apprentissage, ont su s'approprier cette compétence et en maitriser la frontière de prix avec leur délégataire. Cette performance nous semble redevable de l'écriture contractuelle des conventions TER liant la SNCF à leur commanditaire régional. Soulignons notamment le choix des clauses d'indexation des charges d'exploitation SNCF-C1, mais aussi les conséquences du système financier d'incitation à la performance (bonus-malus et pénalités) et enfin le partage du 
risque sur recettes (Desmaris, 2004, 2010a). La question qui se pose dès lors est de savoir s'il est possible d'aller plus avant dans le sens d'une réduction des coûts unitaires. 


\section{Le processus collectif d'apprentissage en région Rhône-Alpes : du couple Région-SNCF au cercle élargi des acteurs du transport}

La région Rhône-Alpes est à l'évidence une région clé du point de vue du transport régional de voyageurs. Rhône-Alpes, c'est 135000 déplacements journaliers en TER en 2009, 250 trains régionaux et 550 cars TER en 2010, $394 \mathrm{M} €$ de budget de fonctionnement en 2010. Cette région est ainsi la plus importante des vingt régions TER, tant du point de vue du trafic régional, avec plus de $18 \%$ du total métropolitain, que du point de vue de la SNCF, avec le plus gros budget TER de France. La Région Rhône-Alpes est aussi une des plus investie, avec un effort budgétaire en faveur du TER parmi les plus élevés de toutes les régions (23,4\% du budget de la Région en 2008).

La politique TER de Rhône-Alpes s'est délibérément voulue ambitieuse, comme le montrent clairement le rythme de modernisation du matériel roulant, le fort volontarisme régional en matière de politique tarifaire, le choix très précoce du cadencement (début en décembre 2007, généralisation en 2009) et les choix de restructuration de l'offre visant à mieux mailler le territoire et à proposer une vraie cohérence de la chaîne de transport centrée sur l'intermodalité (services de rabattement, stationnements adéquats en gare, consignes vélos, horaires coordonnés.... $)^{9}$. Ceci étant, Rhône-Alpes n’a pas cédé à l'activisme de l'offre, à la différence de nombre d'autres régions. Le volume de trains-km offert a augmenté de 12,7\% entre fin 2001 et fin 2007, contre 17,4\% en moyenne (Haenel, 2008).

Au terme de la convention SNCF-TER 2002-2006, les résultats enregistrés par Rhône-Alpes nous semblent s'apparenter à une logique de type « gagnant-gagnant ».

Les voyageurs sont les tout premiers gagnants. Ce résultat est flagrant à la lecture des chiffres de la fréquentation TER, avec une progression de 38,6\% entre fin 2001 et fin 2007 (contre 31\% en moyenne), et plus de 10 à $12 \%$ de fréquentation annuelle pour les meilleures années. L'attractivité du TER Rhône-Alpes est aussi probablement celle qui a connu l'évolution la plus favorable, avec une amélioration du ratio voyageurs-km sur trains-km de plus de $23 \%$, contre 10,3\% en moyenne sur 2001-2007 (Desmaris, 2010a). Cette performance est la récompense tant d'une politique tarifaire particulière imaginative (Cartes de réduction Coup de Cœur, Coup Double, Coup de Foudre et Coup de Pouce) que de l'engagement régional en faveur de la modernisation du matériel roulant.

Gagnante est également la SNCF qui obtient des transferts financiers significatifs dans le cadre du conventionnement TER. Alors que dans presque toutes les régions, le coût moyen des charges d'exploitation forfaitisées s'est réduit en valeur réelle, ce n'est pas le cas en Rhône-Alpes (et en PACA). Le prix unitaire par train-kilomètre réalisé a augmenté de $8 \%$, enregistrant l'essentiel de cette hausse ponctuellement, en tout début de Convention. Par la suite, cette hausse se stabilise, du fait notamment que la convention prévoit un quota d'offre marginale à un coût inférieur de 20\%. Secondairement, la SNCF obtient structurellement un montant «d'héritages " non négligeable au titre de la rémunération de l'exploitant qui, curieusement dans cette région, augmente avec le temps (Desmaris, 2004). Mais la SNCF pourrait être encore davantage gagnante si elle réussissait à améliorer plus significativement la qualité du service produit, et tout particulièrement la régularité et la continuité du service. Les déficiences en la matière, sanctionnées financièrement, lui font reperdre en pénalités une grande partie du bénéfice de ce contrat.

\footnotetext{
${ }^{9}$ Schéma Régional des Services de Transport (SRST) Rhône-Alpes, adopté le 10 avril 2008.
} 
Gagnante, enfin, est la Région (et les contribuables), puisque l'efficience économique de la dépense TER est une de celles qui a enregistré l'évolution la plus favorable. L'augmentation du prix par train-kilomètre de la subvention d'exploitation est maîtrisée, avec une hausse de 2,7\% en rythme annuel moyen sur la Convention (en monnaie courante), et une baisse sur les trois dernières années. Le coût moyen du TER en termes de subvention d'exploitation reste cependant comparativement un peu plus élevé que la moyenne en Rhône-Alpes, du fait du coût des péages (Desmaris, 2010a).

Au total, cette équation « tous gagnants », perceptible en Rhône-Alpes, résulte de la hausse de la fréquentation et donc des gains de productivité conventionnels obtenus. Le cadre conventionnel, subtil et exigeant ${ }^{10}$, a également joué un rôle important, amenant l'ensemble des parties à pouvoir bénéficier des bénéfices de l'apprentissage organisationnel en cours.

Il n’est donc par surprenant que ce résultat ait placé la région dans une position centrale en matière de transport collectif (Haenel, 2008). Dans la mesure où les usagers des trains ont des trajets qui ne commencent ni de finissent dans les gares, une réflexion commune a été engagée avec les responsables des communes et autres autorités organisatrices. Ainsi, à l'échelle de la région urbaine de Lyon, a été lancée l'initiative REAL (Réseau express de l'agglomération de Lyon). Le 22 février 2005, les représentants de la région Rhône-Alpes, du Grand-Lyon, du Sytral, de la SNCF et du Conseil général du département du Rhône signaient un protocole d'accord ambitieux comme en témoignent les extraits suivants :

Le phénomène de périurbanisation bouleverse les distances et les périmètres de déplacements quotidiens des habitants. La modification des usages est plus rapide que la création et l'évolution des différents modes et systèmes de transports publics. Pour répondre à ce défi, il est nécessaire de renforcer l'intégration des réseaux, afin d'offrir un service unifié, performant et facilitant les déplacements. A cette fin son énoncés les principes suivants :

- coordination de l'ensemble des offres de transports publics en particulier le maillage des réseaux existants,

- mise en place de dessertes ferroviaires cadencées,

- mise en place d'une tarification intégrée et de supports de titres de transport unifiés et utilisables dans tous les réseaux de transports,

- adaptation des services aux besoins des personnes à mobilité réduite,

- organisation de rabattement en transports collectifs sur les axes structurants,

- modernisation et création de pôles d'échanges et développement de parkings de rabattement attractifs pour les automobilistes et les cyclistes,

- développement de systèmes d'information communs pour les voyageurs.

Le plus intéressant est que la plupart de ces principes ont été, sinon entièrement concrétisés, du moins largement mis en œuvre. Il en a été ainsi du cadencement ferroviaire et de la tarification intégrée. En outre, des projets spécifiques, cités dans le protocole d'accord, ont été réalisés comme les nouvelles lignes de tramway du Sytral. Le lecteur trouvera aussi sur le site internet MULTITUD un exemple des efforts consentis en matière d'information du voyageur.

\footnotetext{
${ }^{10}$ Avec une méthodologie empruntée à la théorie des contrats, et à partir de l’hypothèse de co-traitance, DESMARIS (2004) qualifiait le conventionnement SNCF-TER de Rhône-Alpes de « rationnel légal » fondé sur un mode de coordination faisant appel à « l’autorité ».
} 


\section{Vers des discontinuités dans le processus d'apprentissage?}

Au vu des résultats présentés dans les pages qui précèdent, l'économiste est en droit de poser une question sur l'efficience du monopole d'exploitation confié à la SNCF. Dans les manuels d'économie, le transport ferroviaire est, au titre des industries de réseau, présenté comme un cas d'école du monopole naturel, fruit de la présence de rendements croissants. Or, en matière de TER, si nous avons bien en France un monopole, celui de la SNCF, on s'interroge sur les rendements croissants. Comme nous venons de le voir, s’il n’y a pas, hors péages, de dérive à la hausse des coûts pour la collectivité, on ne voit pas apparaître réellement cette baisse tendancielle des coûts unitaires que nous décrit la notion de courbe d'apprentissage (Asher, 1956 ; Alchian, 1963). Si cette situation devait perdurer, il faudrait sans doute, pour des raisons financières, limiter la progression de l'offre, ou trouver de nouvelles ressources.

Nous commencerons cette seconde partie par rappeler les incertitudes qui caractérisent les variables clés de l'équation financière du système TER (2.1.). Devant les risques de dérive des coûts pour la collectivité, nous nous intéresserons ensuite aux perspectives offertes par l'ouverture à la concurrence (2.2.). Conformément à certains enseignements de la littérature en matière de " courbe d'apprentissage " pourrait-on observer un décrochement, une baisse sensible des coûts proche de ce qui a été observé dans certains Länder allemands ? Le recours à la concurrence constituerait bien sûr une discontinuité majeure dans le processus collectif d'apprentissage, tout comme le serait une autre option, celle qui remettrait en cause, ne serait-ce que partiellement, l’encouragement systématique à la mobilité (2.3.).

\subsection{Les inconnues du système de financement des TER}

Que pouvons-nous dire de la soutenabilité financière de la politique TER conduite par les régions, tant du point de vue de l'État, des régions, que de celui de la SNCF et de RFF ? L'État a accepté, par les textes de décentralisation, de s'engager financièrement à compenser aux régions le coût du transfert de cette compétence, sur la base du périmètre de ce service pour l'année 2000, afin de sacrifier au principe de neutralité financière ardemment souhaité par les régions. Cette compensation financière visait plusieurs objectifs : couvrir le déficit d'exploitation versé à la SNCF, compenser les tarifications sociales mises en œuvre par l'État, mais aussi permettre la modernisation des matériels roulant, particulièrement vétustes à l'époque. En outre, l'État avait accepté de cofinancer un programme d'investissement destiné à la modernisation des gares à vocation régionale (loi SRU, art. 128).

Les modalités de calcul de cette compensation financière ayant été modifiées depuis 2002, il est difficile aujourd'hui d'en calculer précisément le montant. Reprenant les estimations effectuées par la Commission Transports des comptes de la Nation, nous évaluons ces compensations à 1,9 milliard d'euros en 2009, contre 1,4 milliard en 2002 (un tiers de plus). Ces compensations visent principalement à couvrir le déficit d'exploitation (88\% de la contribution, soit 1,7 milliard). Le poids financier de la modernisation du matériel roulant est donc supporté, pour l'essentiel, par les régions qui en portent l'initiative.

L'effort financier de l'État pour accompagner cette décentralisation est, au vu de ces chiffres, tout à fait considérable. Le sénateur H. Haenel (2008, p. 9) le relevait dans son rapport d'évaluation de la régionalisation ferroviaire, en faisant de ce transfert financier une des principales clés de son succès. 
Tableau 4. Les compensations financières de l’État aux régions pour le TER.

\begin{tabular}{lccccc}
\hline $\begin{array}{l}\text { En millions d'euros } \\
\text { courants }\end{array}$ & 2002 & 2007 & 2008 & 2009 & $\begin{array}{c}\text { Variation } \\
\text { 2002/2009 en \% }\end{array}$ \\
\hline Total & 1439 & 1866 & 1905 & 1905 & $+32,4 \%$ \\
Exploitation & 1439 & 1642 & 1676 & 1676 & $+35,1 \%$ \\
$\quad \begin{array}{c}\text { Contribution pour } \\
\text { l'exploitation des services } \\
\text { transférés aux régions }\end{array}$ & 1071 & 1449 & 1479 & 1479 & $+38,1 \%$ \\
$\begin{array}{c}\text { Dotation de compensation } \\
\text { des tarifs sociaux }\end{array}$ & 170 & 193 & 198 & 198 & $+16,5 \%$ \\
$\begin{array}{c}\text { Investissement } \\
\text { Dotation complémentaire }\end{array}$ & 198 & 224 & 229 & 229 & $+15,7 \%$ \\
\hline $\begin{array}{c}\text { pour le renouvellement du } \\
\text { matériel roulant }\end{array}$ & 198 & 224 & 229 & 229 & \\
\hline
\end{tabular}

Source : Nos calculs d'après Les Comptes des Transports de la Nation 2010 (2009, p. 117).

En dépit de cet effort financier de l’État, le coût net du TER pour les régions, une fois déduits les transferts opérés par l'État au titre des TER s'est particulièrement alourdi ${ }^{11}$, enregistrant une progression sensible, comme l’illustrent nos calculs (Figure 9).

Figure 9. Évolution des contributions nettes (des compensations de l’État) des régions au titre du TER.

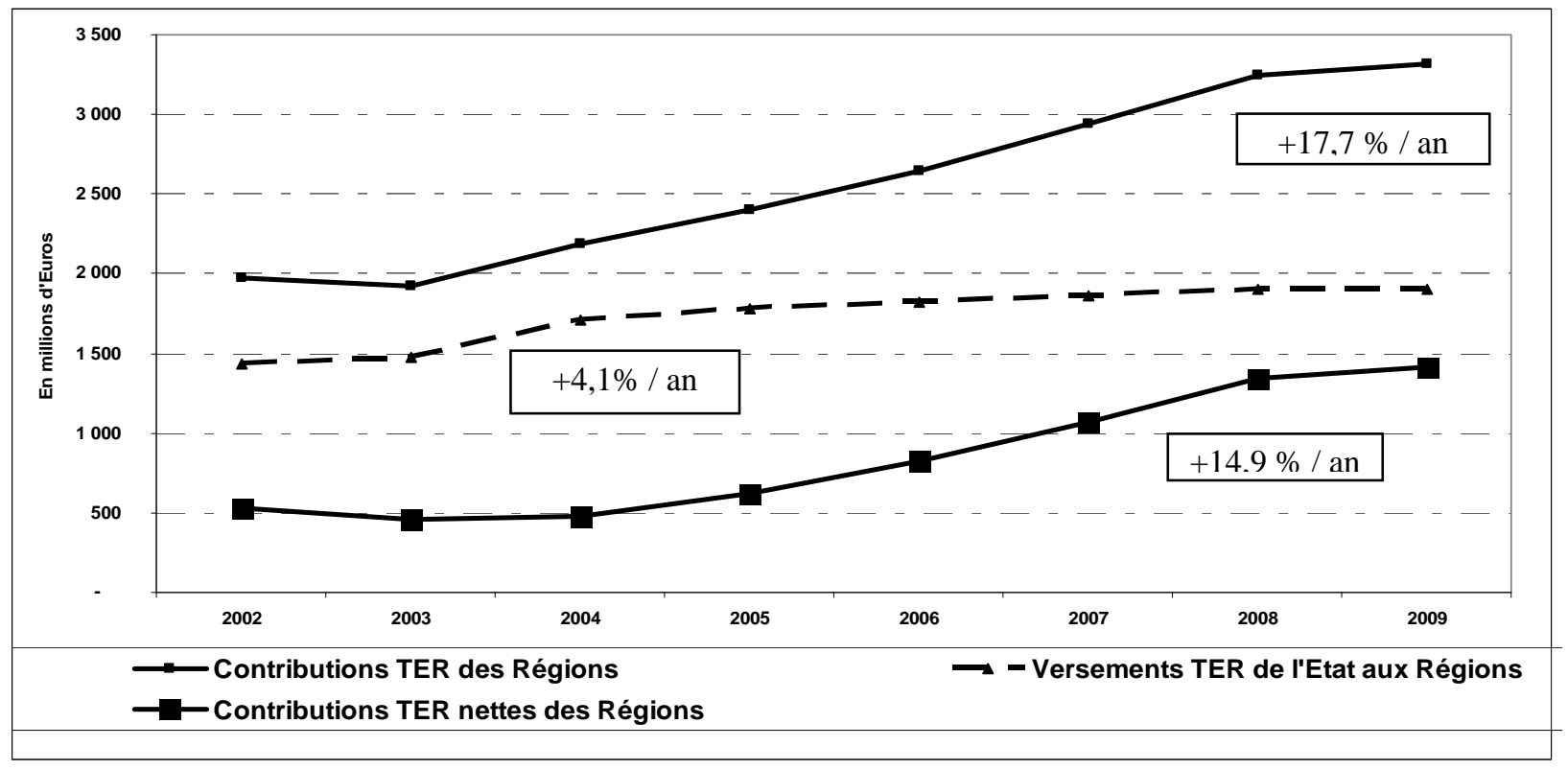

Source : Nos calculs à partir à partir des Comptes des Transports de la Nation 2002, 2005, 2007 et 2010, MEEDDAT/SESP.

\footnotetext{
${ }^{11}$ Cela fait dire à certains Président de Région que la décentralisation (ferroviaire) est un jeu de dupe entre l’État et les Collectivités, puisque décentralisation dit démocratie et proximité, donc respect des usagers et, au final, obligation de financer des améliorations du service offert (PERCHERON, 2004, p. 42).
} 
Globalement, le coût des TER a progressé de 68\%, avec une progression des compensations de l'État aux régions de l'ordre de 32\% mais dans le même temps une multiplication par 2,6 $(+160 \%)$ de la contribution nette des régions. La question de la soutenabilité financière du TER pour les régions est donc ouverte du fait de ce taux de progression annuel moyen, de presque $15 \%$ sur cette période, largement supérieur à celui de la progression des ressources, notamment fiscales des collectivités régionales. Nous observerons cependant que l'année 2009 marque la première inflexion dans cette trajectoire de hausse continue. Est-ce le point d'arrêt de cette spirale?

Selon la Cour des Comptes (2009 p. 43), le bilan financier du TER serait également mitigé et ambigu pour la SNCF. La Cour relevait que « l'activité TER est en expansion mais ne développe pas sa rentabilité ». Plus exactement, si, d'un point de vue commercial, le TER est un succès par sa contribution positive à l'augmentation du chiffre d'affaires du groupe, sa rentabilité financière est par contre faible et aurait eu tendance à décroitre, en tout cas sur la génération des Conventions signées en 2002.

Ainsi, le TER représente 16,8\% du chiffre d'affaires total de la SNCF en 2007, contre 14\% en 2002, soit une progression globale de $42 \%$ sur la période largement supérieure à celles des autres activités de l'EPIC SNCF (Tableau 5). Mais la contribution de l'activité TER à la rentabilité du groupe SNCF est bien plus limitée. Elle n'est en moyenne que de 16 millions d'euros sur la période, c'est-à-dire 3\% du résultat annuel moyen de la SNCF (EPIC). De plus, le taux de marge sur l'activité, faible en niveau, ne fait que se dégrader sur la période (Tableau 5).

Tableau 5. Chiffre d'affaires, excédent brut d'exploitation et résultat net de la SNCF et de son activité TER.

\begin{tabular}{|c|c|c|c|c|c|c|c|}
\hline En millions d'euros & 2002 & 2003 & 2004 & 2005 & 2006 & 2007 & $\begin{array}{l}\text { Variation } \\
2002-2007 \\
\text { en } \%\end{array}$ \\
\hline \multicolumn{8}{|l|}{ SNCF- EPIC } \\
\hline Chiffre d'affaires (CA) & 14782 & 14742 & 15526 & 16009 & 16743 & 17406 & 17,8 \\
\hline $\begin{array}{l}\text { EBE (Excédent brut } \\
\text { d'exploitation) }\end{array}$ & 753 & 784 & 1176 & 1418 & 1487 & 1665 & 121,1 \\
\hline Résultat net & 19 & 50 & 490 & 1334 & 138 & 997 & 5147,4 \\
\hline \multicolumn{8}{|l|}{ Activité TER } \\
\hline Chiffre d'affaires & 2063 & 2137 & 2481 & 2610 & 2778 & 2928 & 41,9 \\
\hline EBE & 128 & 133 & 158 & 132 & 57 & 113 & $-11,7$ \\
\hline Résultat net (RN) & 30 & 35 & 59 & 38 & -47 & -21 & $-170,0$ \\
\hline \multicolumn{8}{|c|}{ Taux de rentabilité activité TER } \\
\hline $\begin{array}{r}\text { Taux de marge en } \% \\
\text { (RN/CA)*100 }\end{array}$ & 1,45 & 1,64 & 2,38 & 1,46 & $-1,69$ & $-0,72$ & $-149,3$ \\
\hline $\begin{array}{r}\text { Taux de marge brute en } \% \\
(\text { EBE/CA })^{\star} 100\end{array}$ & 6,20 & 6,22 & 6,37 & 5,06 & 2,05 & 3,86 & $-37,8$ \\
\hline
\end{tabular}

Source : Nos calculs d'après la Cour des Comptes (2009, p. 42).

Cette dégradation de la rentabilité TER tient à deux facteurs, l'augmentation des charges, supérieure à ce qui est couvert par les régions, et la forte augmentation du montant des pénalités et malus supportés par la SNCF dans l'exécution des conventions, pour qualité de service insuffisante. Ceci étant, à l'instar de la Cour des Comptes, nous serions tentés par une certaine 
réserve à l'égard de ces résultats calculés à partir des informations transmises par la SNCF et non soumise à vérification des commissaires aux comptes ${ }^{12}$.

Le bilan financier du TER pour RFF, le gestionnaire d'infrastructure, pourrait également être moins favorable qu'une première approche pourrait le faire penser. Retenant une lecture en termes de coûts complets, la Cour des Comptes (2009, pp. 45-47) estimait que le TER constituait pour RFF un manque à gagner du fait de l'inadaptation des règles de fixation des péages, très éloignés des coûts complets ${ }^{13}$. En 2008, le taux de couverture des coûts de l'activité TER par les péages d'infrastructure aurait été en moyenne de seulement 32\%, contre 19\% en 2002. Cette amélioration du taux de couverture, sensible pour les régions ${ }^{14}$, reste donc, du point de vue du gestionnaire d'infrastructure, très insuffisante pour assurer l'équilibre économique à long terme de cette activité. Cette tendance ne doit pas dissimuler d'importantes différences entre régions. En 2008, le taux de couverture est de 50\% en Alsace, 46\% en PACA, 42\% en Rhône-Alpes, mais seulement de 35\% dans le Centre, 25\% dans les Pays de la Loire et de 14\% dans le Limousin...

Avec des taux de couverture qui ne dépassent pas 50\%, les fonds publics seront donc durablement mobilisés par l'activité TER. Or, comme les autres collectivités territoriales, et les administrations publiques en général, les régions vont être confrontées à des contraintes financières croissantes dans les années à venir. Cela résulte notamment de l'évolution de la fiscalité et de la base fiscale (Cossardeaux, 2010). Les régions ont récemment (Loi de Finances 2010) perdu toute possibilité d'influer sur le cours des impôts locaux avec la disparition de la part régionale de la taxe foncière et la suppression de la taxe professionnelle, qui constituait une recette « dynamique ». Ces dernières sont désormais remplacées par la contribution économique territoriale (CET) dont l'évolution est à l'initiative de l'État. Aussi, même si les finances régionales restent généralement saines, avec un niveau d'endettement parfaitement soutenable (DGCL), il n'est pas surprenant de constater que le rapport Haenel 2008 envisage de façon détaillées les ressources supplémentaires qui pourraient venir abonder les budgets régionaux.

Ecartant l'hypothèse de l'affectation d'une petite part de la TIPP (taxe intérieure sur les produits pétroliers), fréquemment avancée par un autre sénateur alsacien, Adrien Zeller, alors président de la région Alsace, R. Haenel envisage que le versement transport (VT) puisse être étendu au financement des TER. La démonstration est argumentée : l'histoire du VT n'est-elle pas celle d'un élargissement progressif de sa zone d'application ? Dans la mesure où il a été d'abord appliqué à la seule région parisienne, puis étendu progressivement aux grandes et petites agglomérations (aujourd'hui le seuil est de 10000 habitants !), on comprend bien pourquoi les régions, devenues un acteur central de l'organisation de la mobilité quotidienne, souhaitent également en profiter. La question de la fiscalité est donc importante et la réforme annoncée de l’organisation des collectivités territoriales (nouvelle articulation entre région et départements) va rencontrer ici une question clé.

Le sénateur Haenel évoque aussi une autre source de revenus pour les régions, la hausse du prix payé par l'usager, notamment ceux qui bénéficient d'un abonnement. Cette perspective est évidemment abordée de façon prudente dans la mesure où les capacités contributives ne sont pas très élevées. Même si une partie de la clientèle des TER est aujourd'hui constituée de personnes disposant d'un certain pouvoir d'achat, la discrimination tarifaire est délicate à mettre en œuvre. Faudrait-il par exemple faire varier le prix de l'abonnement travail avec le revenu de la personne, ou du ménage ? Si on voit bien l'intérêt d’un tel mécanisme, sa mise en œuvre ne serait pas

\footnotetext{
${ }^{12}$ L'on sait combien l'imputation des charges de structure dans un groupe peut contribuer à fortement modifier l'affichage de sa rentabilité.

${ }^{13}$ Nous relèverons que l'appréciation de la Cour est effectuée à partir d'une étude de RFF.

${ }^{14}$ Zembri (2005) soulignait alors la crainte des Régions de devoir payer deux fois la maintenance et al remise çà niveau de l'infrastructure, une première fois à travers les péages et une seconde fois, directement, pour suppléer à l'insuffisance du gestionnaire du réseau ferré, et en amont de l’État son unique actionnaire.
} 
simple. D’autant que dans les années passées, les offres tarifaires promotionnelles se sont au contraire multipliées (voir encadré sur la région Rhône-Alpes) pour attirer les clients vers les TER. Si l'on ajoute à cela le fait que de nombreux conseils généraux ont adopté pour leur transport par autocar une tarification plate (2 euros le voyage quelle que soit la distance), on comprend qu'une participation financière accrue des usagers serait une vraie discontinuité, certains diraient un retour en arrière.

En définitive, la régionalisation ferroviaire présente des effets bénéfiques pour les usagers. Mais ce résultat est obtenu au prix d'un fort engagement financier de la Collectivité et présente un bilan économique encore incertain pour la SNCF et pour RFF. Derrière ce que l'on pourrait qualifier de "modèle économique SNCF-TER-SRU », se profilent des disparités régionales particulièrement fortes qui font de chaque Région un modèle économique bien particulier. On peut d'ailleurs noter que même s'il n'existe pas officiellement de concurrence du fait du monopole de la SNCF, chaque région a instauré avec l'exploitant des relations qui expliquent en partie les résultats plus ou moins favorables obtenus (Lévêque, 2006 ; Desmaris, 2010, 2011). On ne peut donc que se féliciter du fait qu'au sein de l'ARF (Association des Régions de France), les échanges d'expérience se développent dans le sens d'un meilleur partage de l'information entre l'autorité organisatrice et la SNCF. Il peut en résulter une rationalisation de l'offre. Mais cela peut-il aller jusqu'à l'acceptation de l'entrée sur le marché de nouveaux opérateurs capables de faire baisser sensiblement le coût pour la collectivité ?

\subsection{Réduire les coûts du TER : vers une ouverture à la concurrence ?}

Dans le secteur ferroviaire, et tout particulièrement en France, la concurrence est une réalité récente. Du fait des évolutions réglementaires européennes, elle existe depuis quelques années pour le transport de marchandises (mars 2006 en France), mais fonctionne plutôt comme un jeu à somme négative en France. Les parts de marché des nouveaux entrants progressent (près de 20\% au début de 2011), mais sans que cela compense la réduction du trafic fret opéré par la SNCF. La part de marché du ferroviaire dans le transport de marchandises continue donc à diminuer en France, après comme avant l'ouverture à la concurrence. En la matière, la France se distingue de la plupart des autres pays européens où l'introduction de la concurrence dans le trafic de marchandises s'est accompagnée d'une croissance soutenue du trafic et d'une légère reprise de part modale, au-moins jusqu'à la crise actuelle.

Pourquoi dès lors s’intéresser à la concurrence dans le transport régional de voyageurs ? La réponse tient au fait que pour les TER, il ne s'agit pas d'une concurrence « sur le marché », mais d'une concurrence " pour le marché » (Crozet, 2004). Dans ce cas de figure, la baisse des trafics est très peu probable puisqu'il y a peu de changements pour les voyageurs. C'est l'autorité organisatrice qui contracte directement avec un ou plusieurs opérateurs pour exploiter tout ou partie des lignes de son territoire. Par le biais d'un appel d'offre, l'autorité organisatrice peut donc fixer un cahier des charges précis concernant le contenu de l'offre ferroviaire (quantité, qualité...) tout en retenant l'exploitant qui demande le plus faible niveau de subventions.

Les expériences conduites dans d'autres pays sont intéressantes, elles montrent que des gains substantiels peuvent être obtenus, débouchant sur un développement sensible de l'offre, pour un coût identique, voire moindre pour la collectivité. S. Séguret (2009) a ainsi présenté des données suggestives en comparant la situation du Land du Schleswig-Holstein (Nord de l'Allemagne) et de la région Rhône-Alpes. Ces deux entités étaient en 2008 relativement comparables en termes ferroviaires : 23,6 millions de trains kilomètres pour la première contre 24,3 pour la deuxième. Mais la comparaison des coûts totaux de fonctionnement donne des résultats beaucoup plus tranchés : 275 millions d'euros pour la première contre plus de 500 millions pour la seconde ! En d'autres termes, le coût d'un train-kilomètre est légèrement supérieur à 20 euros en RhôneAlpes et proche de 12 euros dans le Schleswig-Holstein. Comme le montant total des péages 
acquittés en France est, pour les trafics sous revue, légèrement inférieur à ce qu'il est en Allemagne, la différence se situe essentiellement dans les coûts d'exploitation.

Ainsi que nous l'a expliqué lors d'un entretien, Jochen Schulz, responsable du transport ferroviaire dans ce Land, il ne faut pas chercher l'explication dans un quelconque « downsizing » social synonyme de baisse des salaires des personnels. Les conducteurs de train sont en Allemagne au moins aussi bien rémunérés qu'en France. C'est l'organisation du travail qui est totalement différente. Le temps de conduite par jour est plus élevé, entre 5 et 6 heures ce qui permet aux conducteurs de revenir le plus souvent à leur domicile en fin de service. Cela réduit très fortement les coûts annexes que représentent les primes de "découché » et les jours de repos en récupération. Or, conséquence directe du fameux règlement « RH 077 », ces coûts sont très élevés au sein de la SNCF. Au fil des ans, la gestion des ressources humaines s'est rigidifiée au sein de la SNCF rendant très difficile toute réforme organisationnelle ambitieuse. Or cette réforme est indispensable alors que la SNCF elle-même, dans un rapport interne rendu public par la presse, reconnaissait que pour les TER, ses coûts de fonctionnement sont de $30 \%$ supérieurs à ceux de ses concurrents. Le problème est donc clairement posé : s'il faut baisser les coûts de façon significative, avec ou sans ouverture à la concurrence, il faudra s'attaquer de façon drastique aux sureffectifs de la SNCF et donc aux conventions collectives, temps de travail, gestion des ressources humaines, design des postes de travail... plus qu'une discontinuité, une rupture (Gauthier-Lescop L., Lévêque J., 2006).

Notons aussi qu'un autre mécanisme vertueux a fonctionné en Allemagne: la contrainte budgétaire ! Les élus du Land de Schleswig-Holstein ont donné à leurs services techniques une consigne simple : les améliorations de l'offre ne peuvent se faire qu'à coût constant, au mieux ! L'ouverture à la concurrence a donc joué un rôle important pour inciter les entreprises concurrentes à améliorer leurs propositions et pour offrir aux services techniques la possibilité de réfléchir à une nouvelle organisation. Le cadencement a ainsi été généralisé pour que les graphiques de circulation soient plus homogènes et plus denses. Le réseau du Land a été affecté à 6 opérateurs différents (dont la DB et Veolia, mais aussi une filiale des Chemins de fer Luxembourgeois) qui exploitent soit de façon exclusive certaines lignes, les plus petites, soit de façon partagée, pour les plus importantes. Sur certaines lignes, l'ouverture à la concurrence a permis d'obtenir des baisses de prix significatives, pouvant dépasser les 30\% par train-kilomètre.

Les régions françaises sont donc confrontées à un dilemme, économique, mais aussi politique et social.

- Soit, dans une logique de continuité, elles poursuivent la contractualisation avec la seule SNCF maintenue dans son statut de prestataire unique. Le seul espoir de réduire les coûts réside alors dans l'amplification du processus d'apprentissages en cours. Nous avons ainsi vu que la région Rhône-Alpes a obtenu de la SNCF un rabais de 20\% sur les trains additionnels. Mais, globalement, les gains potentiels seront faibles.

- Soit, n’hésitant pas à miser sur la discontinuité, envisager une rupture partielle plus ou moins significative et prendre l'initiative de faire entrer de nouveaux compétiteurs, dans l'espoir de gains d'efficience très supérieurs. Ce serait inscrire la France dans la voie allemande et dans le sens recommandé par le règlement communautaire de 2007, dit règlement $\mathrm{OSP}^{15}$. Mais cela pose de redoutables questions que le conservatisme ferroviaire français aborde difficilement.

\footnotetext{
${ }^{15}$ Le règlement 1370/2007/CE du Parlement européen et du Conseil du 23 octobre 2007 est relatif aux obligations de service public de transport de voyageurs par chemins de fer et par route. Il est dit règlement OSP.
} 
Ces difficultés ont été présentées dans le rapport du sénateur Grignon ${ }^{16}$, elles renvoient au problème de reclassement du personnel SNCF non repris par le nouvel entrant. Si en effet ce dernier est capable de réduire sensiblement les coûts de fonctionnement, c'est qu'il a besoin de sensiblement moins de personnel que la SNCF pour une offre similaire. En Allemagne, le problème a pu être résolu. D'une part, car la DB a conduit depuis plusieurs années une politique de réduction des effectifs et de hausse de la productivité. D'autre part, car l'ouverture à la concurrence a poussé à un accroissement global des trafics régionaux, y compris pour la $\mathrm{DB}$, qui a pu ainsi opérer de nombreux reclassements internes (Desmaris C., 2010a).

En France, un tel mécanisme vertueux (hausse de la productivité, mais recyclage des effectifs grâce aux hausses de trafic) est délicat à envisager car il suppose que soient acceptées la nouvelle donne économique du secteur et une certaine flexibilité pour les personnels. Il n’est donc pas surprenant que la loi Grenelle 2, qui avait envisagé un temps d'autoriser l'ouverture à la concurrence, ait préféré d'attendre que les directives européennes imposent cette ouverture.

L'ouverture à la concurrence du transport ferroviaire régionale ne se fera pas en France avant le milieu des années 2010, d'autant que d'autres difficultés demeurent, et non des moindres, comme la question de la propriété des matériels roulants, l'accès aux centres de maintenance, la gestion des espaces dans les gares. Franchir tous ces obstacles va aussi demander un long apprentissage !

\subsection{Vers un moindre encouragement à la mobilité ?}

S’il n'est pas possible de réduire sensiblement les coûts unitaires par un recours large à la concurrence, les régions risquent d'être confrontées à des contraintes financières accrues dans les prochaines années du fait d'un effet de ciseaux : le plafonnement des recettes d'une part et la poursuite de l'accroissement de la demande d'autre part. L'actuel jeu d'acteurs est le suivant.

Les salariés de la SNCF comptent sur des améliorations de leurs revenus, mais ils sont rétifs aux gains de productivité. RFF de son côté cherche à accroître ses ressources au gré des travaux et des requalifications des portions de voie, notamment dans les zones urbaines à fort trafic. L'État a transféré les responsabilités et compense les charges correspondant au service au moment du transfert. Mais comme l'État vient d'annoncer un gel pour trois années des dotations aux collectivités territoriales, les régions vont de fait connaître une baisse de leurs ressources en monnaie constante. Cela peut remettre en cause les programmes d'améliorations, plus ou moins incrémentales, y compris le cadencement qui devrait être généralisé en 2012, l'achat de matériels roulants, mais aussi, dans certaines régions, l'attrait pour la grande vitesse. Profitant de lignes à grande vitesse déjà ouvertes ou en projet, dans l'attente aussi de matériels nouveaux (autorail à grande vitesse), plusieurs régions souhaitent procéder à un saut qualitatif de leur offre pour répondre à une demande croissante des usagers.

La grande vitesse deviendrait alors, à l'instar de ce qui se passe déjà entre Lille et Calais, une composante de l'offre TER. Cela pourrait se développer pour les lignes TGV desservant des territoires fortement urbanisés, comme en région PACA. Comme l'a montré le choix du tracé issu du débat public (qui a écarté le trajet le plus court entre Nice et Paris), la tentation est grande de basculer vers un TGV régional, plutôt proche du modèle allemand (Crozet, 2010). A la question du coût de l'infrastructure, à financer en partie par la région, s'ajoutent donc des interrogations sur les services optimaux et la demande qu’ils peuvent générer. Pour que les trafics soient au rendez-vous, faudra-t-il aller jusqu'à subventionner non seulement l'infrastructure, mais aussi l'exploitation, comme pour les TER ? Cela pose des problèmes de soutenabilité environnementale et économique, entre autres.

\footnotetext{
${ }^{16}$ Lors de l'écriture de cet article, ce rapport, maintes fois annoncé, n'est pas encore paru. Une version de travail est néanmoins disponible sur le net. http://www.capital.fr/var/cap/storage/images/media/images/Projet\%20rapport\%20Grignon.pdf
} 
Car si l'offre TER devait inclure une part croissante de grande vitesse, propre à attirer de nombreux anciens utilisateurs de la voiture, le saut qualitatif se doublerait d'un saut quantitatif pour les budgets publics. N'oublions pas que lorsqu'un voyageur bascule de l'automobile vers les transports collectifs, il demande à la collectivité de substituer un travail rémunéré, celui des salariés de la firme de transport, à un travail non rémunéré, celui qu'il effectuait en temps que chauffeur du véhicule. Cette réalité, qui explique les déficits de fonctionnement récurrents des TER, pourrait s'imposer avec encore plus de force si était introduite dans l'offre TER cette discontinuité que représente la grande vitesse pour la mobilité quotidienne.

Au total, le transport ferroviaire régional se trouve aujourd'hui dans une situation paradoxale. Le succès de la première phase de la régionalisation et la progression des trafics qui a été observée appellent le passage à une nouvelle phase, où l'offre TER pourrait être en même temps plus intensive et plus efficiente. Mais cela ne peut se faire en se contentant de prolonger les tendances antérieures. La région Rhône-Alpes s'est essayée à l'exercice dans le cadre d'une prospective baptisé «TER x 4 ». En prolongeant jusqu'en 2025 les tendances du début des années 2000, c'est à une multiplication par 4 des trafics qu'il fallait s'attendre. Même sans aller jusqu'à des progressions aussi élevées, cet exemple montre que la continuité de la réforme appelle des discontinuités dans les pratiques. Or, celles-ci sont marquées par une grande rigidité. Aucun des acteurs ne souhaite vraiment se lancer dans l'inconnu d'une évolution qui demanderait des adaptations exigeantes pour tous. Pour cette raison, il est fort probable que les années 2010 ne seront pas celles d'une offre en forte progression pour les TER. Les contraintes financières pousseront au contraire à remettre en cause le subventionnement accru de la mobilité, notamment des transports collectifs. Cela sera mis sur le compte des difficultés financières, réelles, des administrations publiques. Et cette explication servira de paravent aux autres facteurs, ceux qui résident dans un jeu d'acteurs où l'emporte la préférence pour le statu quo.

\section{Conclusion}

Le transport ferroviaire régional a connu depuis la fin des années 90 une transformation significative. La régionalisation a donné un nouvel élan à une activité qui semblait vouée à se réduire comme une peau de chagrin. Cette évolution est exemplaire, elle montre que les acteurs d'un service public sont à même de progresser dans un processus d'apprentissage collectif. Les résultats positifs de cette dynamique collective sont indéniables, notamment la hausse des trafics en réponse à une amélioration qualitative et quantitative de l'offre. Mais ce succès même appelle à franchir de nouvelles étapes, qui se révèlent être beaucoup plus délicates que les premières, d'autant que se profile une crise des finances publiques, tant à l'échelon régional que national (Haenel, 2008).

A l'aube de la seconde décennie de la régionalisation des TER, tout se passe comme si ce processus avait mangé son pain blanc. Inscrite dans une large continuité, notamment avec l'absence de mise en compétition de l'opérateur historique, l'acte II de la régionalisation contient en germe des discontinuités (la concurrence, la baisse des coûts, hausse ciblé de la tarification...) devant lesquelles la prudence des acteurs en présence ressemble plutôt à de la pusillanimité. 


\section{Bibliographie}

ALCHIAN A., "Reliability of Progress Curves in Airframe production", Econometrica, Vol. 31, N4, 1963, Oct., pp. 679-693.

ASHER H., Cost-Quantity Relationships in the Airframe Industry, Santa Monica, CA: Rand Wright, 1956, July, 202 p.

BARONE S., "Régionalisation des transports collectifs: la fabrication d'une réforme « consensuelle»", Sociologie du Travail, Volume 50, Issue 4, 2008, Octobre-Décembre, pp. 471-488.

BONNET G., THOMÉ B., HOULES L., CANET A., La régionalisation des transports ferroviaires - enseignements de l'expérimentation et perspectives, Rapport d'études du CERTU, CERTU, ENTPE, Université Lyon 2, 2001, 123 p.

CAMPAGNE P.-Y., "Du rapport Nora (1969) au rapport Haenel (1994)", Revue Générale des Chemins de Fer, janvier, n²124, 2004, pp. 15-20.

COSSARDEAUX J., "Les Régions prêtent à dénoncer les conventions TER avec la SNCF", Les Echos, 20/09/2010.

COUR DES COMPTES, Le transfert aux régions du transport express régional (TER) : un bilan mitigé et des évolutions à poursuivre, Rapport public thématique, Paris, Documentation française, 2009, $150 \mathrm{p}$.

CROZET Y., HEROUIN E., "Le transport régional de voyageurs : régionalisation et nouvelles incitations à la performance ferroviaire", Politiques et Management Public, volume XVII, $\mathrm{n}^{\circ} 3$, 1999, pp. 171-193.

CROZET Y., Réformes ferroviaires européennes : à la recherche des "bonnes pratiques », Institut de l'entreprise, Note de benchmarking international, 2004, 72 pages

CROZET Y., "TGV, le temps des doutes ? ", Transport, n 460, 2010, mars-avril, pp. 87-92.

CSSPF, Evaluation de la réforme du secteur du transport ferroviaire, Rapport présenté en novembre 2001, Paris, Documentation Française, 2002, 256 p.

DESMARIS C., "La régionalisation ferroviaire: architecture conventionnelle et modes de gouvernance", Transports, $n^{\circ}$ 424, 2004, mars-avril, pp. 104-115.

DESMARIS C., Le transport ferroviaire régional de voyageurs en France : à la lumière de la théorie néo-institutionnaliste et des comptes de surplus. Thèse de sciences économiques, sous la dir. de Y. Crozet, Université Lyon 2, 2010a, 438 p. + annexes.

DESMARIS C., "La régionalisation ferroviaire en France : une première évaluation par la méthode des comptes de surplus", Revue d'Economie Industrielle, n¹31, 2010b, $3^{\circ}$ Trim., pp. 69-104.

DESMARIS C., "La gouvernance régionale du transport ferroviaire de voyageurs en France : une approche par la méthode des comptes de surplus », Revue d'Economie Régionale et Urbaine, 2011/1, pp. 39-80.

DGCL, 2010, Les comptes des régions, site du Ministère de l'Intérieur et des Collectivités territoriales : http://www.dgcl.interieur.gouv.fr/workspaces/members/desl/documents/finances/

FAIVRE D’ARCIER, B., "Les premiers pas de la régionalisation ferroviaire", Transports, $\mathrm{n}^{\circ}$ 416, 2002, novembre-décembre, pp. 389-397. 
FAURE A., "Doctrine ferroviaire et différenciation régionale : la décentralisation en chantier" dans OLLIVIER-TRIGALO (dir. scientifique), Six régions à l'épreuve des politiques de transport, Collection de l'INRETS, 2007, pp. 34-46.

FAVRE-BULLE E., "L’essor du transport régional de voyageurs", SESP en bref, $\mathrm{n}^{\circ} 3$, janvier, Ministère des Transports, de l'Equipement, du Tourisme et de la Mer, 2006, 4 p.

FITCHRATINGS, "Régionalisation ferroviaire : un futur fardeau pour les régions", Painvin Nicolas et Dumoulin Olivier, 2002, septembre, 6 p.

GAUTHIER-LESCOP L., LEVEQUE J., 2006, "De la régionalisation à la concurrence régulée. Analyse économique et juridique de la future organisation du transport ferroviaire régional de voyageurs", Politiques et Management Public, vol. 24, n¹, pp. 1-28.

GUERANGER D., "La régionalisation ferroviaire - Réflexions sur les enjeux financiers" dans OLLIVIER-TRIGALO (dir. scientifique), Six régions à l'épreuve des politiques de transport, Collection de l'INRETS, 2007, pp. 88-102.

GUIHERY L., "Régionalisation ferroviaire et conventions régions-SNCF : quels enjeux et quelles perspectives ?", Pouvoirs Locaux, n55/III, 2005, pp. 55-60.

HAENEL H., Rapport relatif à l'avenir de la SNCF, Rapport de la commission d'enquête sur la SNCF. N²35, Sénat, Paris, 1993, 2 juin, 271 p.

HAENEL H., Ecrire l'acte II de la révolution ferroviaire régionale, Rapport au Premier Ministre, 2008, 26 octobre, Documentation Française, Paris, 63 p.

HONORE R., "La facture des péages du TGV continue de s’envoler", Les Echos, 15/09/2010.

LEVEQUE J., "Allotissement et rendements d'échelle - Application aux réseaux de transport ferroviaire", Economie et prévision, 2007, 4-5, n¹80-18.

LEVEQUE J., "Analyse économique des chemins de fer français : d'où venons-nous? Où en sommes-nous? Où allons-nous ? ", Sociétal, 2006, n52, pp. 10-16.

Loi 2000-1208 du 13 décembre 2000 relative à la solidarité et au renouvellement urbain, dite loi SRU, JORF du 14 décembre 2000.

Loi 82-1153 du 30 décembre 1982 d'orientation des transports intérieurs (LOTI), JO du 31 décembre 1982.

Loi 97-135 du 13 février 1997 portant création de l'établissement public RFF en vue du renouveau du transport ferroviaire, JO du 15 février 1997.

MINISTERE DES TRANSPORTS, Les Comptes des Transports de la Nation 2009, Tome 1, SOES, Paris, 2010, 145 p.

PERCHERON D., "Le NPC, région pionnière pour le transport collectif ferroviaire", Revue Générale des Chemins de Fer, janvier, n¹24, 2004, pp. 39-45.

SEGURET S., Liberalization of passenger rail transport : new dynamics in French-German outlook, Présention lors de la Summer School "The future of Mobility » organisée par l'université technique de Munich, Frauenchiemsee, Juillet 2009.

SETRA, "Le transport ferroviaire de voyageurs sur le réseau ferré national français - Exploration d'un système complexe", Synthèse des connaissances, Ministère de l'Ecologie, de l'Energie, du Développement Durable et de la Mer, 2009, septembre, 94 p.

STEINMETZ C., "Le conventionnement, un outil au service de la régionalisation ferroviaire", Revue Générale des Chemins de Fer, janvier, n¹24, 2004, pp. 47-56. 
STEINMETZ C., "Régionalisation ferroviaire : bilan et enjeux", Contrats publics, $\mathrm{n}^{\circ} 48$, octobre, 2005, pp. 53-56.

ZEMBRI P., "Vers une seconde étape de la régionalisation des transports ferroviaires : cause et enjeux d'une mutation délicate », Transports urbains, n96, 1997, pp.17-24.

ZEMBRI P., "Régions, État, SNCF : vers une nouvelle crise de confiance ?", Pouvoirs Locaux, n66/III, 2005, pp. 61-64. 